\title{
UNIVERSITYOF BIRMINGHAM

\section{Cooling performance improvement of impingement hybrid synthetic jets in a confined space with the aid of a fluid diode}

\author{
Yu, Qinghua; Mei, Ziyue; Bai, Mengqi; Xie, Danmei; Ding, Yulong; Li, Yongliang
}

DOI:

10.1016/j.applthermaleng.2019.113749

License:

Creative Commons: Attribution-NonCommercial-NoDerivs (CC BY-NC-ND)

\section{Document Version}

Peer reviewed version

Citation for published version (Harvard):

Yu, Q, Mei, Z, Bai, M, Xie, D, Ding, Y \& Li, Y 2019, 'Cooling performance improvement of impingement hybrid synthetic jets in a confined space with the aid of a fluid diode', Applied Thermal Engineering, vol. 157, 113749. https://doi.org/10.1016/j.applthermaleng.2019.113749

Link to publication on Research at Birmingham portal

Publisher Rights Statement:

Checked for eligibility: 24/05/2019

\section{General rights}

Unless a licence is specified above, all rights (including copyright and moral rights) in this document are retained by the authors and/or the copyright holders. The express permission of the copyright holder must be obtained for any use of this material other than for purposes permitted by law.

-Users may freely distribute the URL that is used to identify this publication.

- Users may download and/or print one copy of the publication from the University of Birmingham research portal for the purpose of private study or non-commercial research.

- User may use extracts from the document in line with the concept of 'fair dealing' under the Copyright, Designs and Patents Act 1988 (?)

- Users may not further distribute the material nor use it for the purposes of commercial gain.

Where a licence is displayed above, please note the terms and conditions of the licence govern your use of this document.

When citing, please reference the published version.

Take down policy

While the University of Birmingham exercises care and attention in making items available there are rare occasions when an item has been uploaded in error or has been deemed to be commercially or otherwise sensitive.

If you believe that this is the case for this document, please contact UBIRA@lists.bham.ac.uk providing details and we will remove access to the work immediately and investigate. 


\section{synthetic jets in a confined space with the aid of a fluid diode}

Qinghua $\mathrm{Yu}^{\mathrm{a}, \mathrm{b},{ }^{*}}$, Ziyue Mei ${ }^{\mathrm{b}}$, Mengqi Bai ${ }^{\mathrm{a}}$, Danmei Xie ${ }^{\mathrm{b}}$, Yulong Ding ${ }^{\mathrm{a}}$, Yongliang $\mathrm{Li}^{\mathrm{a}}{ }^{\text {, }}$

${ }^{\text {a }}$ Birmingham Centre for Energy Storage, School of Chemical Engineering, University of Birmingham, Birmingham B15 2TT, United Kingdom Education, Wuhan 430072, China

$22{ }^{*}$ Corresponding author. E-mail address: yqh2015@hotmail.com (Q. Yu)

$23{ }^{\#}$ Corresponding author. E-mail address: Y.Li.1@bham.ac.uk (Y. Li) 


\section{Abstract}

For the conventional synthetic jet in a confined space, the recirculation of heated air at

26 a small nozzle-to-surface spacing results in deterioration of its impingement cooling ability.

27 To overcome this drawback, various novel fluid diodes are designed and introduced to form

28 hybrid synthetic jets in this paper. Numerical analyses are carried out to reveal the cooling

29 performance enhancement mechanism of the hybrid synthetic jets with the aid of fluid 30 diodes. Instantaneous images of velocity contour and streamline at one cycle are 31 comparatively presented to elaborate on their flow characteristics. Based on the simulation 32 results, the volumetric efficiency, air temperature at the nozzle exit, average temperature on 33 the heated surface and average Nusselt number are further calculated to quantify the 34 improvements of the proposed hybrid synthetic jets compared to the conventional synthetic 35 jet. The hybrid synthetic jet with a convergence nozzle generates the best cooling ability 36 among various configurations. This study provides significant guidance for the design of 37 novel hybrid synthetic jets with high-efficiency impingement cooling performance.

39 Keywords: Impingement cooling; Synthetic jet; Fluid diode; Heat transfer; Confined space. 


\begin{tabular}{|c|c|c|c|}
\hline \multicolumn{4}{|c|}{ Nomenclature } \\
\hline \multicolumn{2}{|c|}{ Roman letters } & \multirow[t]{2}{*}{$u_{\max }$} & \multirow{2}{*}{$\begin{array}{l}\text { maximum ejecting velocity at the } \\
\text { nozzle exit during one cycle }\left(\mathrm{m} \cdot \mathrm{s}^{-1}\right)\end{array}$} \\
\hline$A$ & amplitude $(\mu \mathrm{m})$ & & \\
\hline$D_{c}$ & cavity diameter $(\mathrm{mm})$ & $U(t)$ & moving speed of diaphragm $\left(\mu \mathrm{m} \cdot \mathrm{s}^{-1}\right)$ \\
\hline$D_{h}$ & heated surface diameter $(\mathrm{mm})$ & $V_{e}$ & air volume ejected out through the \\
\hline$D_{i}$ & inlet outer diameter $(\mathrm{mm})$ & & nozzle exit in one cycle $\left(\mathrm{m}^{3}\right)$ \\
\hline$D_{n}$ & nozzle exit diameter $(\mathrm{mm})$ & $V_{S}$ & air volume sucked back through the \\
\hline$D_{o}$ & orifice diameter $(\mathrm{mm})$ & & nozzle exit in one cycle $\left(\mathrm{m}^{3}\right)$ \\
\hline$f$ & oscillation frequency $(\mathrm{Hz})$ & $X(t)$ & displacement of diaphragm $(\mu \mathrm{m})$ \\
\hline$h_{c}$ & heat transfer coefficient $\left(\mathrm{W} \cdot \mathrm{m}^{-2} \cdot \mathrm{K}^{-1}\right)$ & & \\
\hline$H$ & nozzle-to-surface spacing $(\mathrm{mm})$ & Greek & letters \\
\hline$H_{c}$ & cavity height $(\mathrm{mm})$ & $\eta_{V}$ & volumetric efficiency \\
\hline$H_{i}$ & inlet chamber height $(\mathrm{mm})$ & $\theta$ & local time-averaged temperature $\left({ }^{\circ} \mathrm{C}\right)$ \\
\hline$H_{n}$ & nozzle length (mm) & $\lambda$ & thermal conductivity $\left(\mathrm{W} \cdot \mathrm{m}^{-1} \cdot \mathrm{K}^{-1}\right)$ \\
\hline$H_{o}$ & orifice plate thickness $(\mathrm{mm})$ & $\mu$ & dynamic viscosity $(\mathrm{Pa} \cdot \mathrm{s})$ \\
\hline$L$ & whole target surface diameter (mm) & $\rho$ & density $\left(\mathrm{kg} \cdot \mathrm{m}^{-3}\right)$ \\
\hline$N u$ & Nusselt number & & \\
\hline$q$ & heat flux on heated surface $\left(\mathrm{W} \cdot \mathrm{m}^{-2}\right)$ & Subscr & \\
\hline$R e$ & Reynolds number & $a$ & ambient air \\
\hline$t$ & time $(\mathrm{s})$ & avg & average \\
\hline$T$ & oscillation period of diaphragm (s) & $c$ & heated surface center \\
\hline$u(t)$ & average velocity at the orifice $\left(\mathrm{m} \cdot \mathrm{s}^{-1}\right)$ & $s$ & heated surface \\
\hline$U_{e}$ & equivalent velocity at the orifice $\left(\mathrm{m} \cdot \mathrm{s}^{-1}\right)$ & & \\
\hline
\end{tabular}




\section{Introduction}

With the developing trends of electronic devices toward miniaturization and high degree of integration, high thermal power density and small space make effective heat removal become more challenging in the design of electronic systems [1]. Heat accumulation will lead to the failure of electronic devices and shorten their service life [2, 3]. Ingenious cooling schemes are absolutely essential to achieving effective thermal management of electronic devices to keep their temperatures within a suitable range. A promising cooling technology, called an impingement synthetic air jet, has gradually attracted widespread attention [4-7], due to its obvious advantages as follows compared with conventional cooling methods, such as natural convection, fan, steady jet: (1) a synthetic jet exhibits higher heat transfer capacity, which can be 4.5-10 times [8], 1.2-1.4 times [9] and 1.4 times [10] higher than those of natural convection, fan and steady jet, respectively; (2) it has no rotating parts, which are conducive to noise reduction, low energy consumption, stability, safety and reliability; (3) it does not need fluid pipe and complex pumping, offering a compact structure and ease of integration. Based on the above advantages, an impingement synthetic air jet is considered to be a more efficient manner for cooling of electronic devices, especially in limited space.

An impingement synthetic air jet is commonly generated by a cavity which contains a small orifice on one sidewall and a periodically oscillating diaphragm mounted on the other sidewall [11]. In virtue of the periodic oscillation of the diaphragm with a certain frequency, the surrounding air is alternately sucked into and ejected from the cavity through the orifice.

61 During the suction stroke, the near-wall low momentum air is entrained into the cavity through the orifice acting as a sink. During the ejection stroke, a coupled vortex pair is generated from the orifice in each cycle and moves toward a heated surface which needs to

64 be cooled. There is no net mass addition but net momentum is injected into the surrounding 
stagnant air. During continuous sucking-ejecting cycles, a fluid jet comprising a train of vortex pairs is formed and consecutively impinges to the heated surface. Hence, the resulting impingement synthetic jet can remove the heat and cool the heated surface. The synthetic jet velocity at the orifice can be estimated by a pressure-based model [12], a lumped element model [13] and a general reduced-order model [14]. The Reynolds number of synthetic jets can be expressed as [15]

$$
R e=\frac{\rho U_{e} D_{o}}{\mu}=\frac{\rho D_{o} L_{o}}{\mu T}
$$

where $\rho$ denotes the air density; $U_{e}$ denotes the equivalent average velocity at the orifice of the cavity; $D_{o}$ denotes the orifice diameter; $\mu$ denotes the air dynamic viscosity; $T$ is the oscillation period of the diaphragm; and $L_{o}$ is the stroke length, which is defined as

$$
L_{o}=\int_{0}^{T / 2} u(t) d t
$$

where $u(t)$ is the time-dependent average velocity at the orifice of the cavity.

Much work has been devoted to the heat transfer characteristics and cooling performance improvement of impingement synthetic air jets. Chaudhari et al. [16] experimentally performed a comprehensive parametric study on heat transfer characteristics of impingement synthetic air jets, including oscillation frequency $(f=100-350 \mathrm{~Hz})$, orifice diameter $\left(D_{o}=5-14 \mathrm{~mm}\right)$, and nozzle-to-surface spacing $\left(H / D_{o}=1-25\right)$. The heat transfer ability of impingement synthetic jets achieved the maximum at the resonance frequency $(f=250 \mathrm{~Hz})$ of the cavity. Small nozzle-to-surface spacing $\left(H / D_{o}<6\right)$ strengthened the confinement effect of the orifice plate and thus got more heated air recirculated between the orifice plate and the heated surface, causing a significant reduction in the heat transfer ability. They subsequently examined the effects of orifice shape [17] and numbers [18]. It was reported that a rectangular orifice with an aspect ratio of 3-5 provided better performance than a square or circular orifice at $H / D_{o} \leq 5$. The configuration of a center orifice surrounded 
by multiple satellite orifices gave better heat transfer performance than the conventional configuration of a single center orifice. Lee et al. [19] numerically studied the interaction of multiple synthetic jets with cross-flow in a micro-channel for the cooling microchip. Double synthetic jets promote the flow mixing in the micro-channel and thus enhance the heat transfer. Rylatt et al. [20] proposed to add ducting to enhance heat transfer of a confined impinging synthetic air jet. Their experimental results indicated that the cold air from a remote location was drawn into the jet flow with the aid of ducting during the suction stroke and the heat transfer was thus improved. Nevertheless, it is not easy to fit ducting in such a small space. Bhapkar et al. [21] experimentally demonstrated that the cooling performance at a small nozzle-to-surface spacing $\left(H / D_{o}<2\right)$ was markedly affected by the orifice cavity confinement and designed various cavity shapes to provide different confinement conditions. The average Nusselt number for least confinement was $44 \%$ higher than that for maximum confinement at $H / D_{o}=0.5$. However, the robustness of the improvement effect provided by

100 the least confinement configuration was not good enough. Liu et al. [22] also reported the

101 fundamental study concerning characteristics of flow and heat transfer of a synthetic air jet under different experimental parameters, such as actuation frequency (200-800 Hz), jet Reynolds number (500-1300), and nozzle-to-surface spacing $\left(H / D_{o}=2-25\right)$. It was found that the highest heat transfer coefficient was achieved using an actuation frequency of $600 \mathrm{~Hz}$ at a nozzle-to-surface spacing of 15 . In addition, when the plate was close to the jet hole

$106\left(H / D_{o}<5\right)$, the heated air was obviously entrapped and recirculated within the small space, which consequently weakened heat transfer. They further proposed to adopt a diffusionshaped orifice to enhance synthetic-jet heat transfer in their succeeding work [23]. It was stated that the diffusion-shaped orifice with an opening angle of $60^{\circ}$ produced a highest heat transfer enhancement of approximately $30 \%$ with respect to a round orifice. 
111 The above researches imply that the principal drawback of the cooling technology

112 based on impingement synthetic jets is inevitable recirculation of the heated air in confined

113 spaces especially when the nozzle-to-surface spacing is small, which leads to relatively poor

114 cooling performance. Integrating fluidic diodes into the actuator of a conventional synthetic

115 jet, which generates a so-called hybrid synthetic jet, is a potentially useful solution for this

116 issue. With the rectification effect of fluidic diodes, the hybrid synthetic jet can entrain

117 amounts of air from the cold surroundings far away from the heat exchange zone, i.e.

118 introduce fresh air without preheating via fluidic diodes into the cooling process. The

119 volumetric efficiency, which is defined as the ratio of the net output fluid volume ejected

120 from the orifice over the whole period to the total fluid volume exchanged through the same

121 orifice over the same period, is used to quantify the rectification performance of the actuators

122 of hybrid synthetic jets [24]. Trávníček et al. [25] designed a hybrid synthetic jet actuator

123 equipped with a pair of fluidic diodes shaped as conical ducts, which offered a volumetric

124 efficiency of $13 \%$. Subsequently, the volumetric efficiency was improved to $19 \%$ by

125 introducing double-acting principle [26] and $70 \%$ by integrating two extremely complicated

126 fluidic diodes [27]. Trávníček et al. [28] tested the impingement heat transfer performance of

127 the hybrid synthetic jet actuator presented in [25] using the heat/mass transfer analogy in

128 unconfined spaces. They reported that it offered a $26 \%$ larger flow rate than the conventional

129 synthetic jet and thus resulted in an increase of $18 \%$ in the heat transfer rate. Hsu et al. [29]

130 designed a double-acting hybrid synthetic jet and experimentally demonstrated that it offered

131 higher vorticity compared with commonly used synthetic jets. They further improved the

132 geometrical configuration of the double-acting hybrid synthetic jet actuators, achieving

133 volumetric efficiencies of 76\% 79\% [30]. Although the impingement hybrid synthetic jet is a

134 promising cooling technology, its detailed heat transfer characteristics and suppression effect

135 on the recirculation of heated air in confined spaces remain largely unaddressed in the 
136 literature. Further, the volumetric efficiency of impingement hybrid synthetic jets

137 theoretically can be up to $100 \%$ like classical reciprocating pumps. Therefore, there is still

138 plenty of room for improvement in volumetric efficiency.

139 This paper designs various novel hybrid synthetic jet actuators equipped with very

140 simple fluidic diodes, aiming to achieve quite high volumetric efficiency and preferable

141 impingement cooling performance. Since numerical simulations based on computational fluid 142 dynamics (CFD) are able to provide precious physical insights and effective prediction

143 without any time-consuming manufacturing [31-33], a full-domain numerical model of heat

144 transfer is established to capture the flow and heat transfer characteristics of impingement

145 synthetic jets in confined spaces. The drawback of the flow structure and the cooling

146 performance at a small nozzle-to-surface for a conventional impingement synthetic jet are

147 first explored as a baseline for comparison. The volumetric efficiencies of the designed

148 hybrid synthetic jet actuators are then predicted whilst the suppression effects of the resulting

149 impingement hybrid synthetic jets on the recirculation of heated air in confined spaces are

150 examined. The cooling performances on a heated surface with a constant heat flux are

151 evaluated in the form of target temperature and Nusselt number. Special attention is paid to

152 the evolution of instantaneous velocity and temperature distribution for all proposed synthetic

153 jets in this paper. This work can provide an essential reference for the proper design of hybrid

154 synthetic jet actuators to gain preferable performance in cooling applications.

155

156 2. Numerical methodology

157 2.1. Numerical model and solving methods

158 The geometry schematic of a typical actuator generating an axisymmetric impingement

159 synthetic jet in a confined space is shown in Fig. 1(a), which is similar to the setup in the

160 work of Persoons et al. [34]. The actuator comprises a cylindrical cavity equipped with an 
161 orifice and an oscillating diaphragm. With the periodic oscillation of the diaphragm, the synthetic jet is formed and impinges on the target surface via the orifice and the nozzle. The target surface is heated with a constant heat flux, which is marked by the red line. The distance between the nozzle exit and the heated surface is named as the nozzle-to-surface spacing. The nozzle exit is labeled as "Nozzle" for the sake of simplicity. The heated surface is embedded in a confined space like a channel. In this paper, the main dimensions of the geometry are set as follows: the orifice diameter $D_{o}=4 \mathrm{~mm}$; the cavity diameter $D_{c}=7.5 D_{o}$ and the cavity height $H_{c}=2.5 D_{o}$; the nozzle length $H_{n}=D_{o}$; the nozzle-to-surface spacing $H$

$169=4 D_{o}$; the heated surface diameter $D_{h}=10 D_{o}$; and the whole target surface diameter $L=$ $20 D_{o}$. Air is selected as the working fluid and the temperature of ambient air is set to $25^{\circ} \mathrm{C}$. flux of $5000 \mathrm{~W} / \mathrm{m}^{2}$ is specified on the heated surface. All other solid walls are set as the adiabatic boundary. Since the outlet section of the channel surrounded by the confined walls

174 is connected to the ambient air, the pressure boundary is adopted there and the outlet static pressure is equal to the atmospheric pressure. The air can freely flow out of and into the channel through the outlet section and the backflow air temperature is set to $25^{\circ} \mathrm{C}$. The noslip boundary for air flow is applied to all solid walls. The oscillation process of the diaphragm can be simplified to a reciprocating movement like a piston with a sinusoidal displacement profile [35]. The time-dependent displacement of the diaphragm is thus defined as [36]

$$
X(t)=A \sin (2 \pi f t)
$$
$\mu \mathrm{m}$ and $f$ is set to $250 \mathrm{~Hz}$. By taking the derivative of $X(t)$, the moving speed of the diaphragm can be derived as

$$
U(t)=2 \pi f A \cos (2 \pi f t) .
$$


186 This equation is used as the boundary condition at the diaphragm of the cavity, which is 187 superior to the flow velocity inlet boundary condition adopted in [15, 37]. This is because the 188 latter imposes fresh air at the inlet within every cycle and cannot allow for the recirculation 189 of hot air, especially at a small nozzle-to-surface spacing. Moreover, the boundary condition 190 adopted in this paper requires less computational time and resources compared with the 191 oscillating membrane boundary condition [36].

192

193

194

195

196

197

198

199

200

201

202

203

204

205

206 


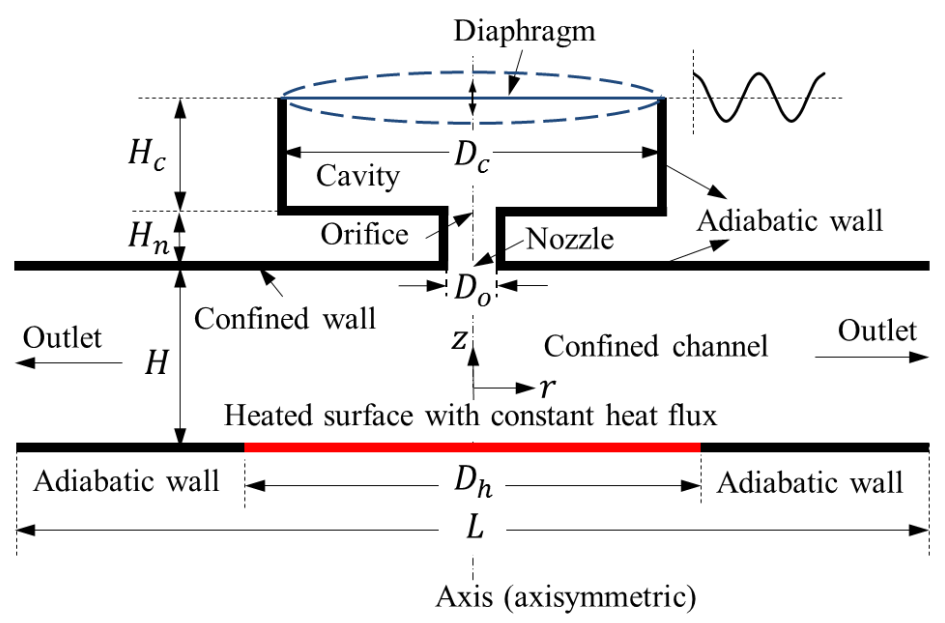

(a)

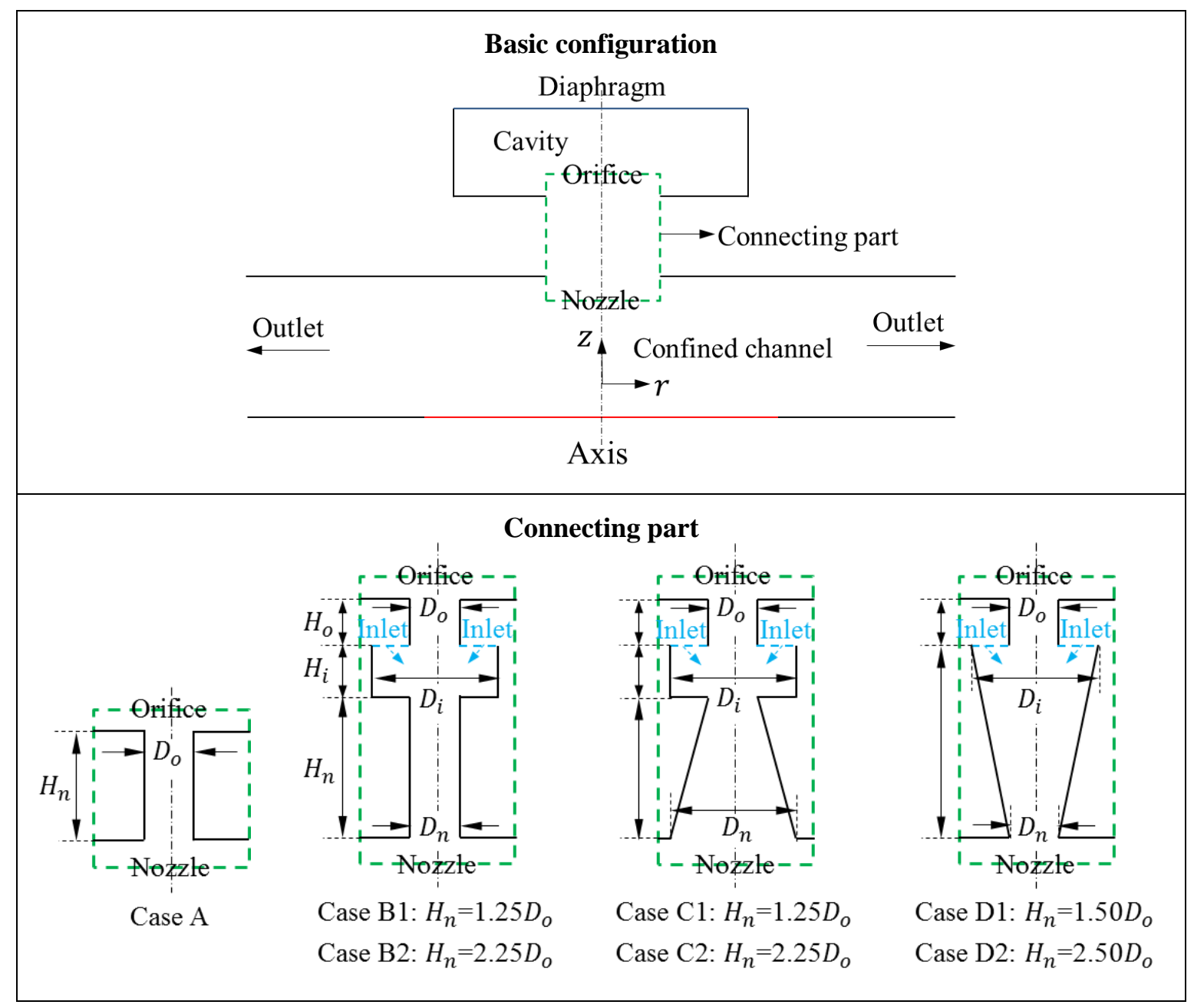

211 Fig. 1 Geometry schematic of (a) a confined impingement synthetic jet actuator and (b) various cases of

212 hybrid synthetic jet actuators based on fluid diodes. 
215 configuration, as shown in the upper part of Fig. 1(b), remains unchanged except the

216 connecting part between the cavity and the confined channel for all cases. As presented in

217 the lower part of Fig. 1(b), Case A is a conventional synthetic jet actuator, which serves as

218 the baseline case; other cases are self-designed hybrid synthetic jet actuators, where so-called

219 fluid diodes are integrated into the connecting parts. The fluid diodes possess an inlet, which

220 is also connected to the ambient air and where the air can freely flow in and out. They still do

221 not require the fluid transport pipeline. The pressure boundary condition is thus applied to the

222 inlet section. All solid walls in the connecting part are assumed to be adiabatic. Case B1 and

223 Case B2 have the same configuration with a cylindrical nozzle and different values of $H_{n}$;

224 Case $\mathrm{C} 1$ and Case $\mathrm{C} 2$ have the same configuration with a divergent nozzle and different 225 values of $H_{n}$; Case D1 and Case D2 have the same configuration with a convergent nozzle 226 and different values of $H_{n}$. The dimensions of the connecting parts for all cases are gathered 227 in Table 1.

Table 1 The dimensions of the connecting parts for all cases.

\begin{tabular}{lllllll}
\hline \multirow{2}{*}{ Case } & \multicolumn{5}{c}{ Dimensions (mm) } \\
\cline { 2 - 5 } & $D_{o}$ & $D_{i}$ & $D_{n}$ & $H_{o}$ & $H_{i}$ & $H_{n}$ \\
\hline $\mathrm{A}$ & 4 & - & - & - & - & $D_{o}$ \\
$\mathrm{~B} 1$ & 4 & $1.5 D_{o}$ & $D_{o}$ & $0.5 D_{o}$ & $0.25 D_{o}$ & $1.25 D_{o}$ \\
$\mathrm{~B} 2$ & 4 & $1.5 D_{o}$ & $D_{o}$ & $0.5 D_{o}$ & $0.25 D_{o}$ & $2.25 D_{o}$ \\
$\mathrm{C} 1$ & 4 & $1.5 D_{o}$ & $1.5 D_{o}$ & $0.5 D_{o}$ & $0.25 D_{o}$ & $1.25 D_{o}$ \\
$\mathrm{C} 2$ & 4 & $1.5 D_{o}$ & $1.5 D_{o}$ & $0.5 D_{o}$ & $0.25 D_{o}$ & $2.25 D_{o}$ \\
$\mathrm{D} 1$ & 4 & $1.5 D_{o}$ & $D_{o}$ & $0.5 D_{o}$ & - & $1.5 D_{o}$ \\
$\mathrm{D} 2$ & 4 & $1.5 D_{o}$ & $D_{o}$ & $0.5 D_{o}$ & - & $2.5 D_{o}$ \\
\hline
\end{tabular}



assumed that the resulting synthetic jets are turbulent for all cases in this study. Actually, the flow regimes of synthetic jets are determined by Reynolds number, which can be calculated by Eq. (1). According to the analysis in Section 3.2, the calculated Reynolds numbers based

234 on the simulation results are 3820 for all cases, which justify the initial turbulent assumption 235 in this study.

The governing equations of mass conservation, momentum conservation and energy conservation for the impingement synthetic jet in the above geometry can be expressed as

$$
\begin{gathered}
\frac{\partial u_{i}}{\partial x_{i}}=0 \\
\rho \frac{\partial u_{i}}{\partial t}+\rho \frac{\partial u_{i} u_{j}}{\partial x_{j}}=-\frac{\partial p}{\partial x_{i}}+\frac{\partial}{\partial x_{i}}\left[\mu\left(\frac{\partial u_{i}}{\partial x_{j}}+\frac{\partial u_{j}}{\partial x_{i}}\right)-\rho \overline{u_{i}^{\prime} u_{j}^{\prime}}\right] \\
\frac{\partial T}{\partial t}+u_{i} \frac{\partial T}{\partial x_{i}}=\frac{\partial}{\partial x_{i}}\left(\alpha \frac{\partial T}{\partial x_{i}}-\overline{u_{i}^{\prime} T^{\prime}}\right)
\end{gathered}
$$

238 where $u_{i}$ is the velocity; $\rho$ is the density; $p$ is the pressure; $\mu$ is the dynamic viscosity; $u_{i}^{\prime}$ is 239 the velocity fluctuation; $T$ is the temperature; $\alpha$ is the thermal diffusivity; and $T^{\prime}$ the 240 temperature fluctuation. In light of the Boussinesq approximation, $-\rho \overline{u_{i}^{\prime} u_{j}^{\prime}}$ is named the 241 turbulent stress and $-\overline{u_{i}^{\prime} T^{\prime}}$ is named turbulent heat flux, which are defined as

$$
\begin{gathered}
-\rho \overline{u_{i}^{\prime} u_{j}^{\prime}}=\mu_{t}\left(\frac{\partial u_{i}}{\partial x_{j}}+\frac{\partial u_{j}}{\partial x_{i}}\right)-\frac{2}{3} \rho k \delta_{i j} \\
-\overline{u_{i}^{\prime} T^{\prime}}=\alpha_{t} \frac{\partial T}{\partial x_{i}}
\end{gathered}
$$

242 where $\mu_{t}$ is the turbulent eddy viscosity; $k$ is the turbulent kinetic energy; and $\alpha_{t}$ is the 243 turbulent diffusivity. 

transfer of the impingement synthetic jet in this paper. The governing equations of mass, momentum and energy conservation are solved within the axisymmetric geometries under cylindrical coordinates as presented in Fig. 1. The air is considered to be an ideal gas with constant thermophysical properties $[15,31]$. The pressure-based transient solver is adopted. The SST/ $k-\omega$ turbulence model is applied to capture the turbulent flow features of the impingement synthetic jet, which has proven to be more suitable than other models and has been repeatedly used in the literature $[15,35]$. The pressure-velocity coupling is achieved by pressure-implicit with splitting of operators (PISO) algorithm. The least squares cell based method was applied to solve the variable gradient at each control volume center. The convective terms, diffusion terms transient terms are discretized with the QUICK scheme, central difference scheme and first-order implicit scheme, respectively. The convergence criterion is set as that the normalised residuals of mass, momentum and energy equations decrease below $10^{-5}, 10^{-5}$ and $10^{-8}$ at each time step, respectively.

In view of the axisymmetric configuration, only a half of the region on the meridian plane is selected as the computational domain, which has been repeatedly adopted to capture the main flow and heat transfer characteristics of impinging synthetic jets in the literature [36, 37]. Further, Hatami et al. [31] and Miró et al. [32] carried out 3D simulations respectively and their results demonstrated distinctly axisymmetric characteristics of impinging synthetic

263 jets under axisymmetric geometrical configurations. Hence, the use of a 2D axisymmetric 264 model can obtain reasonable results with saving calculation resources. The computational domain is meshed totally by non-uniform structured quadrilateral cells for Case A as presented in Fig. 2(a). The computational grids within the connecting parts for Cases B1, C1 and D1 are shown in Fig. 2(b), which are all structured. The grid distributions maintain as similar as possible for different geometrical configurations. The grids near all the solid walls 
are refined to capture large gradients of the velocity and temperature. The sizes of grids

270 adjacent to the walls ensure $\mathrm{y}^{+}<1$. The dynamic mesh technology is used to achieve the

271 oscillation of the diaphragm. Since the grids of the entire computational domain are

272 structured, the grid regeneration during the motion of the diaphragm is accomplished by the

273 layering mesh method, which only affects the gird distribution near the diaphragm and can

274 offer decent grid quality in the entire domain. The user-defined function (UDF) is applied to

275 encode the moving speed of the diaphragm in Eq. (4), which is then compiled and embedded

276 it into Fluent 18.0 as the motion profile of the diaphragm in the dynamic mesh setup to

277 control its moving speed.

278

279

280

281

282

283

284

285

286 


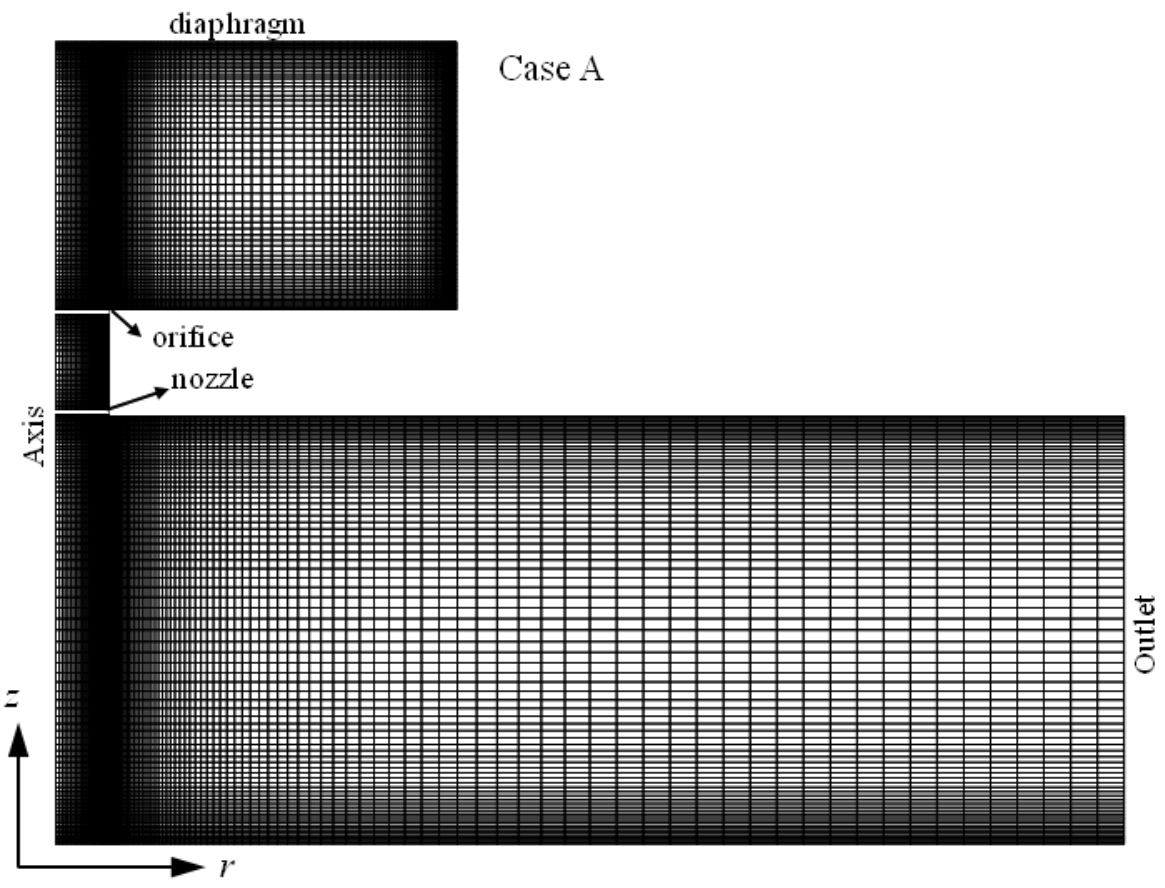

(a)
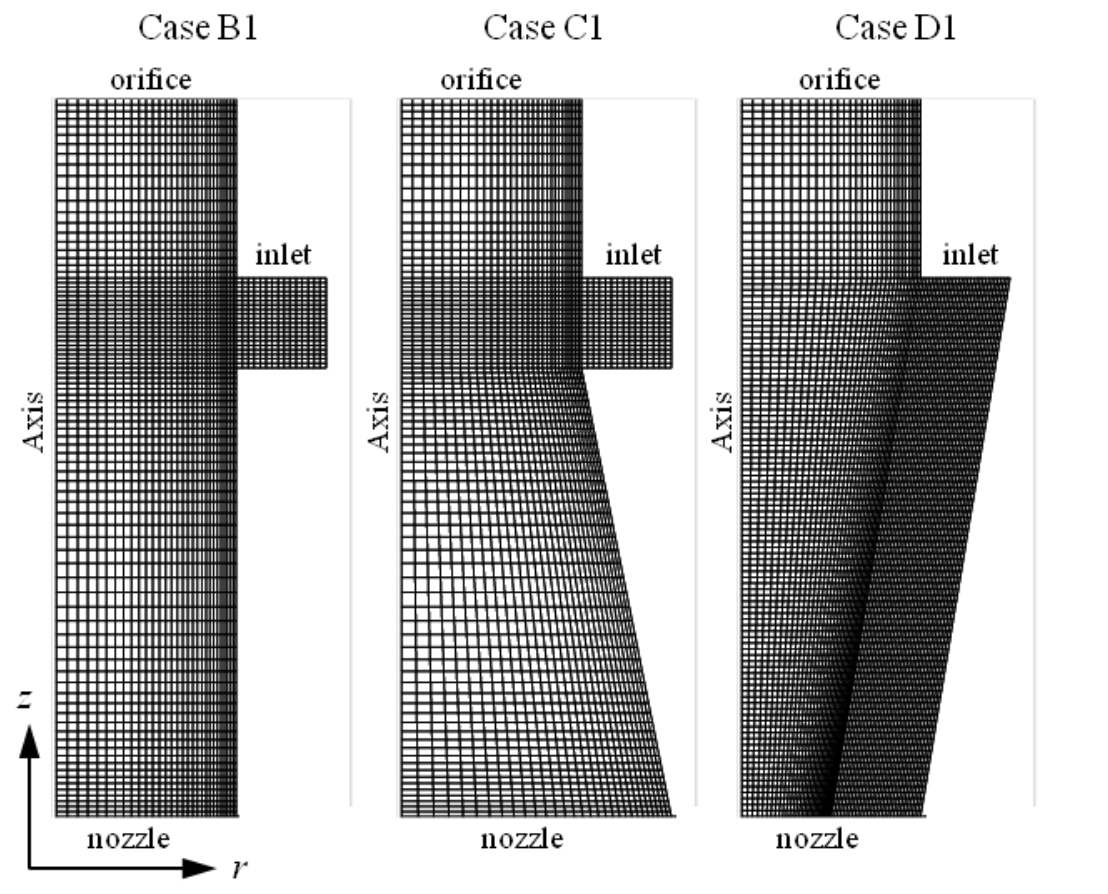

(b)

291 Fig. 2 Computational grids of (a) the entire domain for Case A and (b) connecting parts for Cases B1, C1 292 and D1.

\subsection{Calculation of dimensionless parameters}

The integration of fluid diodes is designed to rectify oscillating air flow to suppress

296 the backflow of hot (heated) air through the nozzle exit in the suction stroke. The volumetric 
297 efficiency is thus introduced to evaluate the rectifying effect of the proposed hybrid synthetic

298 jet actuators on the air flow, which can be defined as [30]

$$
\eta_{V}=\frac{V_{e}-V_{s}}{V_{e}+V_{s}}
$$

299

300 calculated by

$$
N u=\frac{h_{c} D_{o}}{\lambda}=\frac{q D_{o}}{\left(\theta_{s}-\theta_{a}\right) \lambda}
$$

304 where $h_{c}, \lambda, q, \theta_{s}$ and $\theta_{a}$ are the convective heat transfer coefficient, thermal conductivity, constant heat flux on the heated surface, local time-averaged temperature of the heated surface and ambient air temperature, respectively. The average Nusselt number $\left(N u_{\text {avg }}\right)$ can be obtained by replacing $\theta_{s}$ in Eq. (11) with the area-weighted average of $\theta_{s}$ across the heated surface. with about $5000,10,000,20,000,30,000$ cells were adopted to examine the grid independence. Table 2 summarizes the predicted local time-averaged temperature at the heated surface center, maximum ejecting average velocity at the nozzle exit during one cycle and average Nusselt number across the heated surface under the four grid sets. It is obvious that the relative deviation of the fourth grid set $(30,000$ cells) from the third grid set $(20,000$

\subsection{Independence study of grid and time step}

In order to acquire adequately reliable simulation results, independence studies of grid and time step were implemented by taking Case A as an example. Four different grid sets cells) is tiny in all the three variables, which is enough to justify the grid independence when 
the grid cell number is greater than 20,000. For obtaining a trade-off between calculation accuracy and cost, the third grid set (20,000 cells) was thus selected to carry out the further

321 simulations in this study. The choice of the time step should ensure that Courant-Friedrichs-

322 Lewy (CFL) number is less than 1 to maintain numerical stability. Based on the average jet

323 velocity and the mesh size adopted in this paper, the time step should be less than $5 \times 10^{-5}$ (i.e.

$324 T / 80$ ). Comparative analysis among four different time steps of $T / 100, T / 200, T / 500$ and $T / 1000$ was carried out to determine adequate time step. The results in Table 3 demonstrated that the relative deviation of the average Nusselt number between $T / 1000$ and $T / 500$ is enough small and therefore the time step of $T / 500$ is adopted for all simulations.

Table 2 The local time-averaged temperature at the heated surface center $\left(\theta_{c}\right)$, maximum ejecting average velocity at the nozzle exit during one cycle $\left(u_{\max }\right)$ and average Nusselt number $\left(N u_{a v g}\right)$ for Case A under different grid sets.

\begin{tabular}{llllllc}
\hline \multirow{2}{*}{$\begin{array}{l}\text { Grid cell } \\
\text { number }\end{array}$} & \multicolumn{2}{l}{ Predicted values } & \multicolumn{3}{l}{ Relative Deviation from the third grid set (\%) } \\
\cline { 2 - 7 } & $\theta_{c}\left({ }^{\circ} \mathrm{C}\right)$ & $u_{\max }(\mathrm{m} / \mathrm{s})$ & $N u_{\text {avg }}$ & $\theta_{c}$ & $u_{\max }$ & $N u_{\text {avg }}$ \\
\hline 5,000 & 63.1 & 45.9 & 12.21 & 3.52 & 3.85 & 4.36 \\
10,000 & 64.5 & 45.0 & 11.95 & 1.38 & 1.81 & 2.14 \\
20,000 & 65.4 & 44.2 & 11.70 & - & - & - \\
30,000 & 65.6 & 44.0 & 11.64 & 0.31 & 0.46 & 0.51 \\
\hline
\end{tabular}

332

Table 3 Average Nusselt number $\left(N u_{\text {avg }}\right)$ for Case A under different time steps.

\begin{tabular}{lll}
\hline Time step & $N u_{\text {avg }}$ & $\begin{array}{l}\text { Relative Deviation from the third } \\
\text { time step set (\%) }\end{array}$ \\
\hline$T / 100$ & 11.23 & 4.02 \\
$T / 200$ & 11.51 & 1.62 \\
$T / 500$ & 11.70 & - \\
$T / 1000$ & 11.72 & 0.17 \\
\hline
\end{tabular}


338 through two steps. In the first step, the flow characteristic of a synthetic jet predicted by the

339 adopted numerical methods was validated based on the same geometry and boundary

340 conditions as the work of Jian et al. [36]. Fig. 3(a) compares the instantaneous air velocities

341 at $1 \mathrm{~mm}$ away from the nozzle exit along the axis during one cycle predicted by the current

342 model with the corresponding experimental and numerical results obtained by Jian et al. [36].

343 It is evident that they exhibit the similar velocity profile trend and quite minor difference at

344 each instant, which justify a good conformity. In the second step, the heat transfer

345 performance of impingement synthetic jets predicted by the adopted numerical methods in

346 the current model was validated based on the same geometry and boundary conditions as the

347 work of Chaudhari et al. [16]. As shown in Fig. 3(b), the average Nusselt numbers predicted

348 by the current model are compared with those obtained by the experimental work of

349 Chaudhari et al. [16] for $H / D_{o}=2-20$ at $R e=1150$ and $R e=4180$. It can be found that the

350 present results show an appropriate agreement with the experimental data obtained by

351 Chaudhari et al. [16] with a relative difference of less than $\pm 15 \%$ for all combinations of $352 H / D_{o}$ and $R e$. The above comparisons in the two steps demonstrate that the numerical 353 methods adopted in the present model are feasible and valid. 
357

358

359

360

361

362

363

364

365

366

367

368

369

370

371

372

373

374

375

376

377

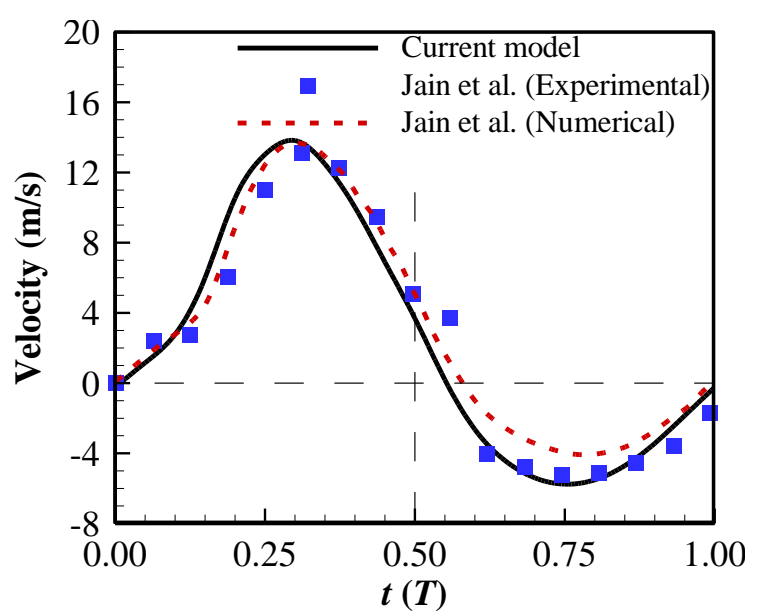

(a)

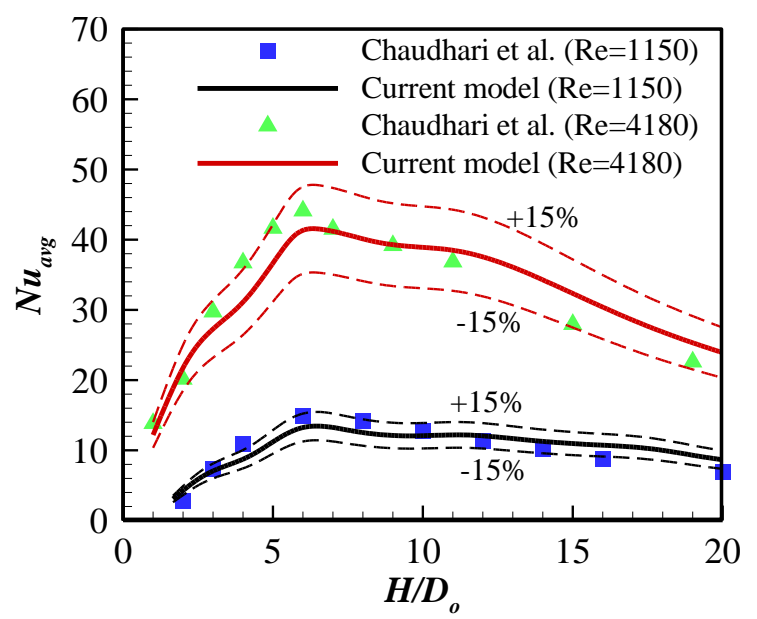

(b)

Fig. 3 Model validation: (a) comparison of instantaneous velocity with the experimental and numerical results of Jian et al. [36]; (b) comparison of average Nusselt number with the experimental results of Chaudhari et al. [16].

\subsection{Analysis of flow characteristics}

By monitoring instantaneous velocities at $0 \mathrm{~mm}$ and $1 \mathrm{~mm}$ away from the center of the nozzle exit, it is found that the monitored velocities become stable only over three cycles at the same oscillation phase of the diaphragm, i.e. the periodicity of velocity variation is achieved only after three cycles. The results are in line with the work of Jain et al. [36] and Xia et al. [37]. Therefore, the analyses on the flow characteristics and heat transfer performances are carried out after three cycles. When the oscillating diaphragm is at the bottom end, i.e. the ejection stroke is just finished and the sucking stroke is about to start, we assumed that a cycle is initiated.

According to the preliminary calculations in this study, it is found that the cooling performance of the impingement synthetic jet notably deteriorated as $H / D_{o}<6$. Theferfore, the case with $H / D_{o}=4$ is taken as an example of a small nozzle-to-surface spacing in this paper. Fig. 4 shows the streamlines and velocity contours for Case A with $H / D_{o}=4$ and $H / D_{o}=8$ at four typical instants of one cycle. As observed in Fig. 4(a) for Case A with $H / D_{o}$ $=4$, the flow in the cavity is dominated by a large clockwise vortex, while the flow in the 
channel is dominated by an anticlockwise large vortex in the entire cycle. When the diaphragm moves from the bottom end to the neutral position $(t=0 T-0.25 T)$, the average sucking velocity near the orifice gradually increases from zero to the maximum. A volume of air is sucked into the cavity through the orifice and incorporated into the large clockwise vortex. Some fresh air flows into the channel through the lower outlet section, which partially flows out of the channel through the upper outlet section directly and partially flows into the cavity along the large anti-clockwise vortex inside the channel and the upper confined wall.

This part of air is heated by the large anti-clockwise vortex before flowing into the cavity although it is helpful for decreasing the air temperature near the orifice. When the diaphragm moves from the neutral position to the top end $(t=0.25 T-0.50 T)$, the average sucking velocity near the orifice gradually decreases to zero and the sucking stroke ends. The two large vortices within the cavity and the channel are gradually decoupled and eventually become independent of each other. Meanwhile, the amount of the fresh (i.e. cold) air sucked into the cavity is progressively decreased to zero. Eventually, all the fresh air flowing into the channel through the lower outlet section immediately turns back near the outlet and directly flows out of the channel via the upper outlet section, which means the net flow rate at the outlet section is zero. When the diaphragm moves from the top end to the neutral position $(t=$ $0.50 T-0.75 T)$, the average ejecting velocity near the orifice gradually increases from zero to the maximum. A volume of air is ejected from the cavity to the channel, which induces the generation of a small anticlockwise vortex near the nozzle exit. The vortex coexists with the anticlockwise large vortex and moves downstream towards the heated surface. The heated air partially flows out of the channel along the heated surface through the outlet section and partially stays within the channel in the form of the anticlockwise large vortex. When the diaphragm moves from the neutral position to the top end $(t=0.75 T-1.00 T)$, the average ejecting velocity near the orifice gradually declines to zero and the ejection stroke ends. The 
amount of air flowing out of the channel along the heated surface also reduces to zero

404 gradually. The flow structure tends to be similar to that at $t=0.50 T$. The moving direction of

405 the small induced vortex changes from towards the heated surface along the axis to towards

406 the outlet along the heated surface. More specifically, the small vortex is generated near the

407 orifice, moves downwards and impinges on the heated surface, and then move along the

408 heated surface towards the outlet, and eventually vanishes. The specific evolution route of the small vortex is 1-2-3-4-5-6 as marked in Fig. 4(a). It implies that the small vortex marked by 5 is evolved from that marked by 1 generated in the previous cycle. When it moves to the

411 position marked by 6 , it is incorporated into the large anti-clockwise vortex and vanishes.

412 Most of the air is recirculated in the confined space and only very little amount of fresh air is

413 supplemented into the confined space in one cycle. It means that the heated (i.e. used) air is repeatedly sucked into and ejected from the cavity. This leads to the deterioration of heat transfer or cooling performance, which will be discussed in the later section.

As observed in Fig. 4(b) for Case A with $H / D_{o}=8$, the flow structure in the cavity is

417 similar to that for $H / D_{o}=4$, while that in the channel is totally different from the latter. The

418 flow in the channel is no longer dominated by a large anti-clockwise vortex, which is a replaced by C-shape flow structure. There is always fresh air flowing into the channel through the upper outlet section as the supplement in the entire cycle. The fresh air is sucked into the cavity along the upper confined wall or incorporated into the jet ejected from the cavity to impinge on the heated surface. Meanwhile, the heated air continuously flows out of the channel via the lower outlet section in the entire cycle along the heated surface. It implies that the heat generated at the heated surface is endlessly transported to the outside of the channel by the involved cold air for $H / D_{o}=8$ and thus the heated surface is effectively cooled. Comparison of flow structures between Fig. 4(a) and Fig. 4(b) demonstrates that the 
427 small nozzle-to-surface spacing $\left(H / D_{o}=4\right)$ restricts the supplement of the fresh air and the 428 discharge of the heated air.

429

430
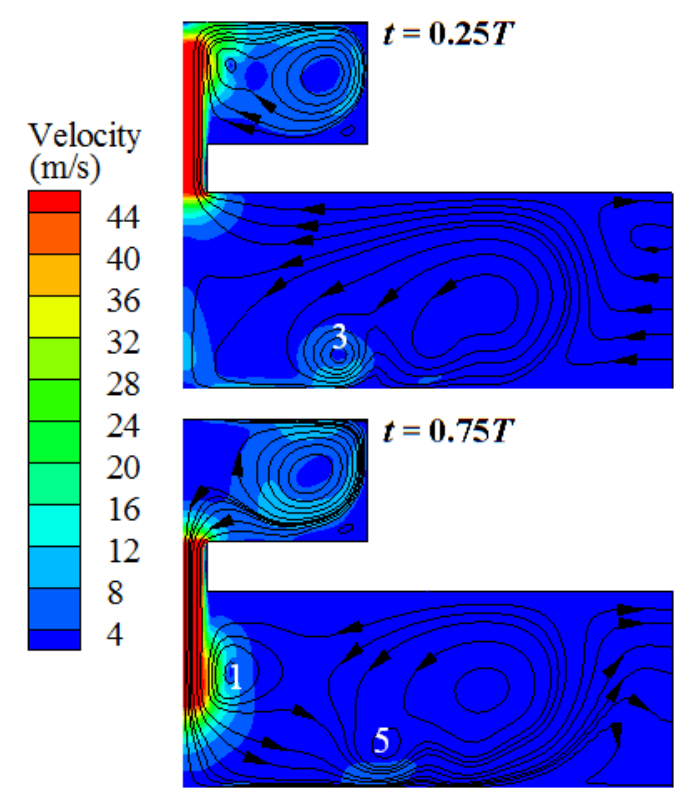

(a)

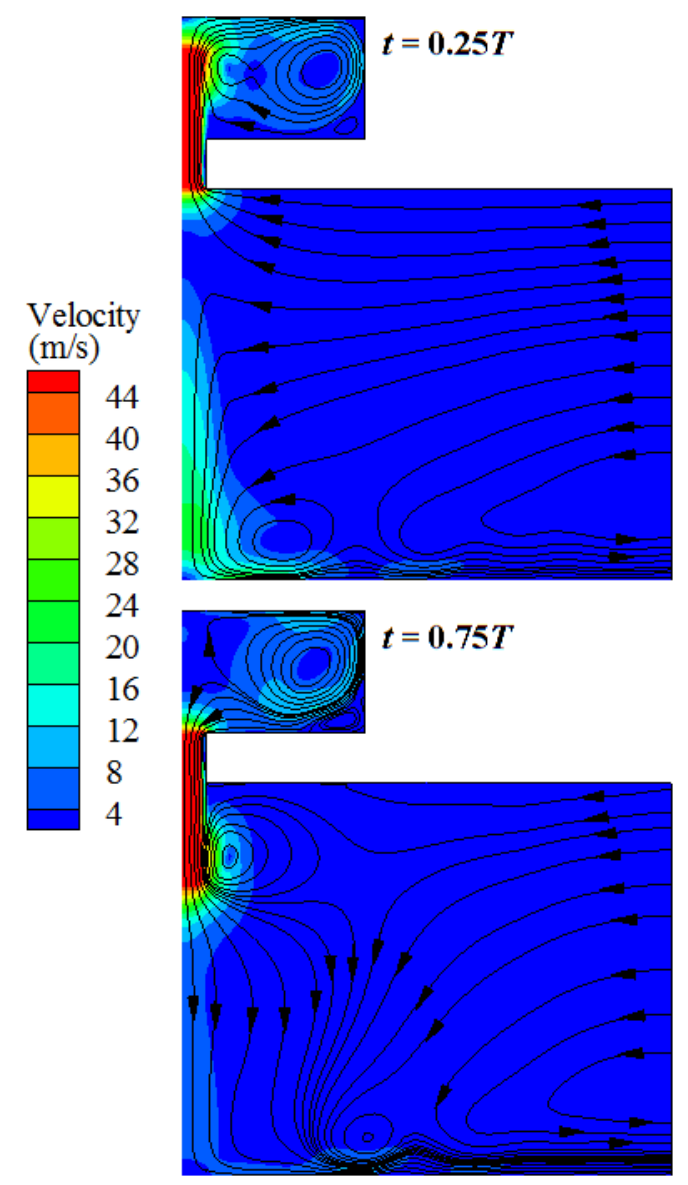

(b)
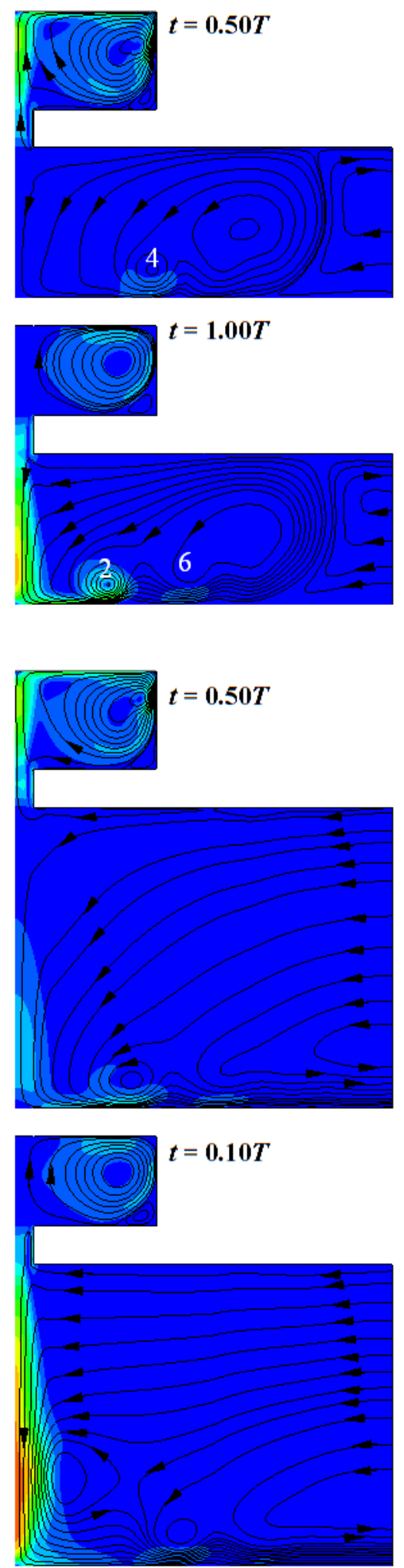

431

432

433 Fig. 4 Velocity contours and streamlines for Case A with (a) $H / D_{o}=4$ and (b) $H / D_{o}=8$ at four different 434 instants of one cycle. 
To overcome the above-mentioned drawbacks of Case A with the small nozzle-tosurface spacing $\left(H / D_{o}=4\right)$, various hybrid synthetic jets based on fluidic diodes were

437 designed. In the following study, all cases have $H / D_{o}=4$. Fig. 5 presents velocity contours and streamlines of hybrid synthetic jets at four typical instants for Case C2 and Case D2 as examples. Compared with Case A as shown in Fig. 4(a), the improvements in Case C2 as

440 illustrated in Fig. 5(a) are as follows: In the whole suction stroke $(t=0 T-0.50 T)$, there is 441 some fresh air continuously flows into the cavity through the lower outlet section, and plenty of fresh air is supplemented into the cavity via the inlet of the connecting part. In the whole ejection stroke $(t=0.50 T-1.00 T)$, plenty of air is ejected from the cavity and impinges on the heated surface, which also induces some fresh air from the inlet to impinge on the heated surface at the same time; Further, a part of the heated air continuously flows out of the channel through the upper outlet section. More improvements are provided by Case D2 as illustrated in Fig. 5(b), which are as follows: In the whole cycle, the air nearly maintains the unidirectional downward flow in the nozzle, except that the nozzle is blocked by a vortex at the end of the suction stroke. It means that plenty of cold fresh air is continuously supplied via the inlet of the connecting part and some air from the cavity and/or the inlet continuously impinges on the heated surface. These improvements are attributed to the combined action of the inlet and the convergent nozzle. The inlet can supplement the fresh air freely. The convergence nozzle can speed up the air flowing downwards, while it serves as a diffuser for the air flowing upwards and thus slows down the air. It means the convergence nozzle exhibits large flow resistance for the air flowing upwards. From the above, the proposed cases solve the drawbacks of Case A by promoting the supplement of the cold fresh air and the discharge of the heated air, i.e. suppressing the recirculation of the heated air in the confined space. This is the main mechanism of cooling performance enhancement. 
459

460

461

462

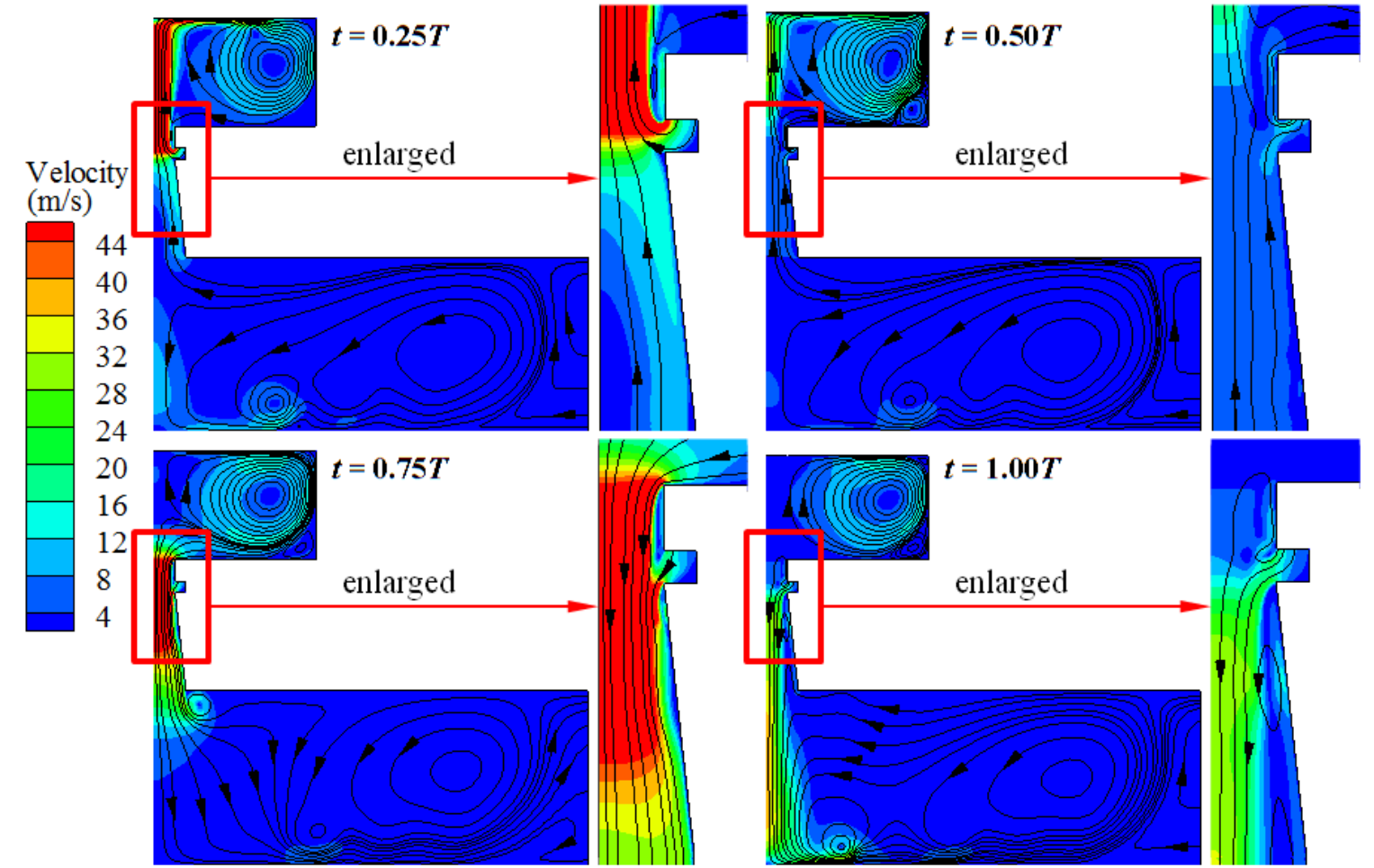

(a)

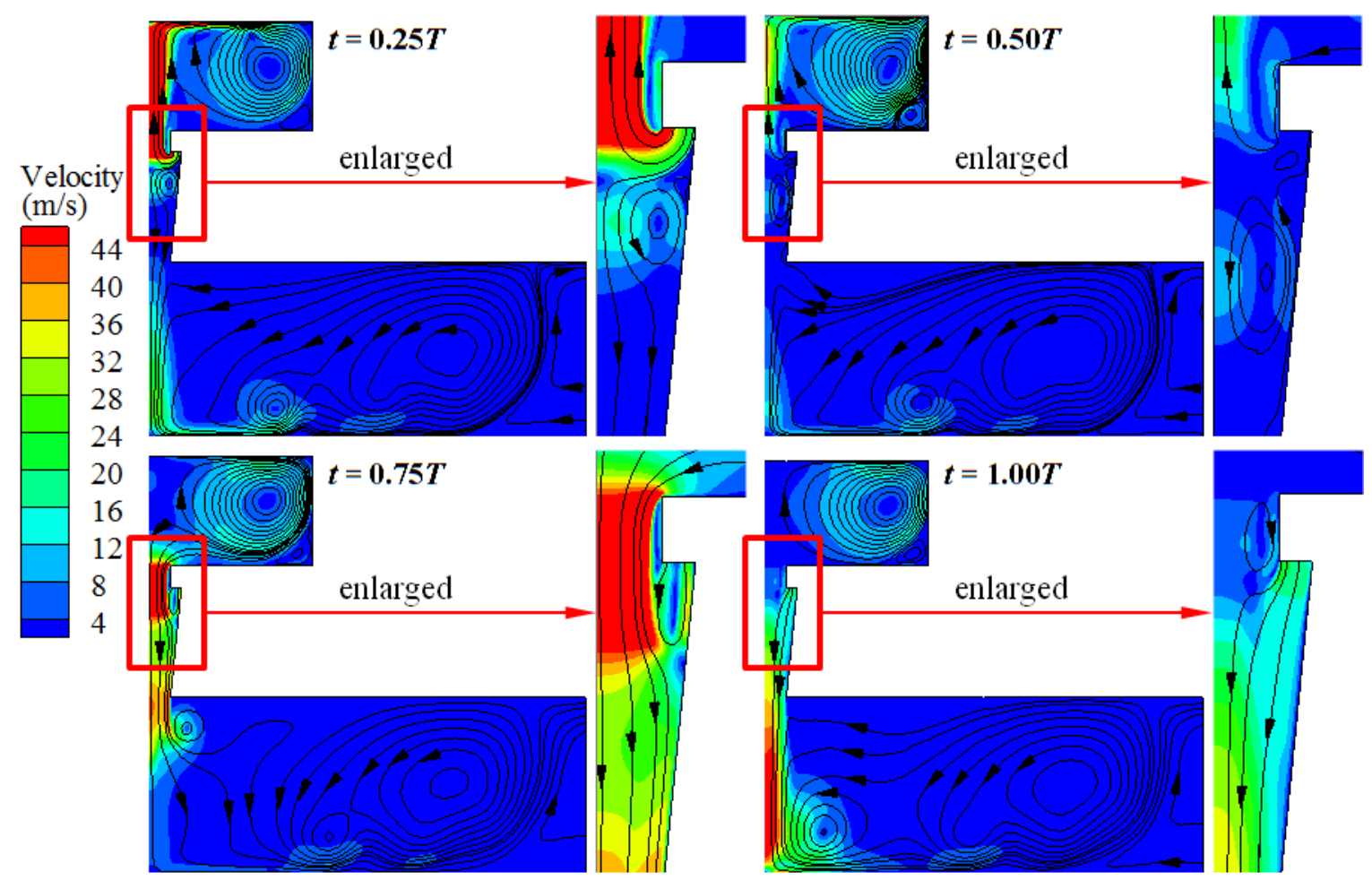

(b)

463 Fig. 5 Velocity contours and streamlines with partially enlarged views for (a) Case C2 and (b) Case D2 at 464 four typical instants of one cycle. 
To quantify the flow characteristics, Fig.6 illustrates the variation curves of area-

467

468

469

470

471

472

473

474

475

476

477

478

479

480

481

482

483

484

485

486

487

488

489

490

averaged velocities with time at the orifice, inlet, outlet and nozzle exit for all cases. It can be seen from Fig. 6(a) that the time-dependent average velocity curves at the orifice nearly keep the same for all cases, which is in line with the similar flow structures in the cavity for all cases as shown in Figs. 4-5. The oscillation of the velocities for all cases is subject to the sinusoidal function, which is consistent with the oscillation of the diaphragm. According to Eq. (1) and Eq. (2), the calculated Reynolds numbers and dimensionless stroke length $\left(L_{o} / D_{o}\right)$ for all cases are 3820 and 14.3, respectively. As presented in Fig. 6(b), the fresh air flows into the cavity or the channel via the inlet in most instants of one cycle except for a small time interval in $t=0.45 T-0.80 T$ for all cases. In this time interval, the air flows out via the inlet. Case D2 offers the maximum instantaneous velocity while Case C1 offers the minimum instantaneous velocity at the inlet among all cases in one cycle. Moreover, the inlet velocity increases with $H_{n}$ under the same geometry configuration. By comparing Fig. 6(b) and Fig. 6(a) for Case D2, it can be found that the flow-in velocity at the inlet is first greater than and then lower than but still close to the velocity flowing into the cavity in the suction stroke. It implies that most of the air in the cavity is supplemented by the inlet and a part of the fresh air from the inlet impinges on the heated surface via the nozzle in the suction stroke, which is in line with Fig. 5(b). As shown in Fig. 6(c), the oscillation of the velocity at the outlet for Case A is still subject to the sinusoidal function, whereas those for other cases deviate from the sinusoidal function. Since the area of the outlet section is the same for all cases, it is evident that less air flows into the channel via the outlet section for all the cases based on fluid diodes compared to Case A in one cycle. The amount of air flowing into the channel via the outlet section for Case D2 is the least among all cases, which is close to zero. It can be found from Fig. 6(d) that the oscillation curve shape of the velocity at the nozzle exit is similar to that at the outlet for all the cases except for Case $\mathrm{C} 1$ and $\mathrm{C} 2$. This is because 
the diameter of the nozzle exit in these two cases is larger than those in other cases as demonstrated in Fig. 1(b). For better comparison, the time-dependent velocity curves in Fig. 6(d) are changed to the time-dependent volume flow rate curves in Fig. 7(a).

494

495

496

497

498

499

500

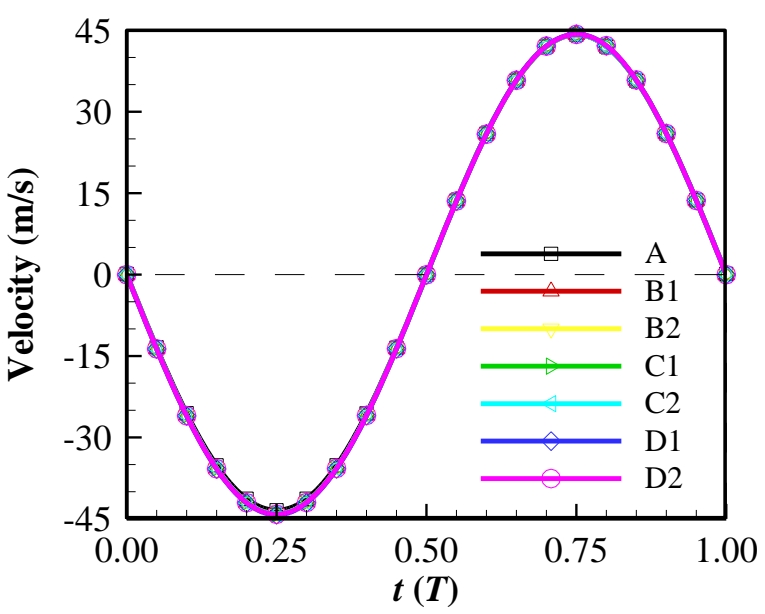

(a)

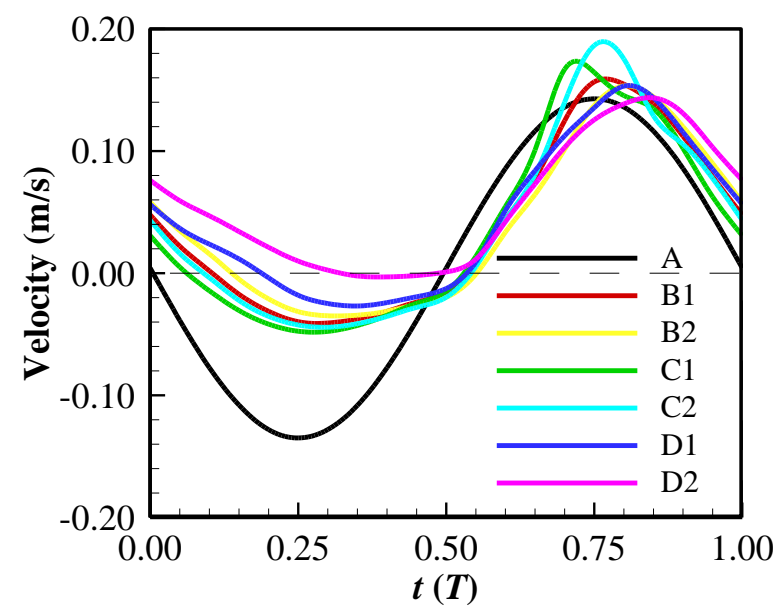

(c)

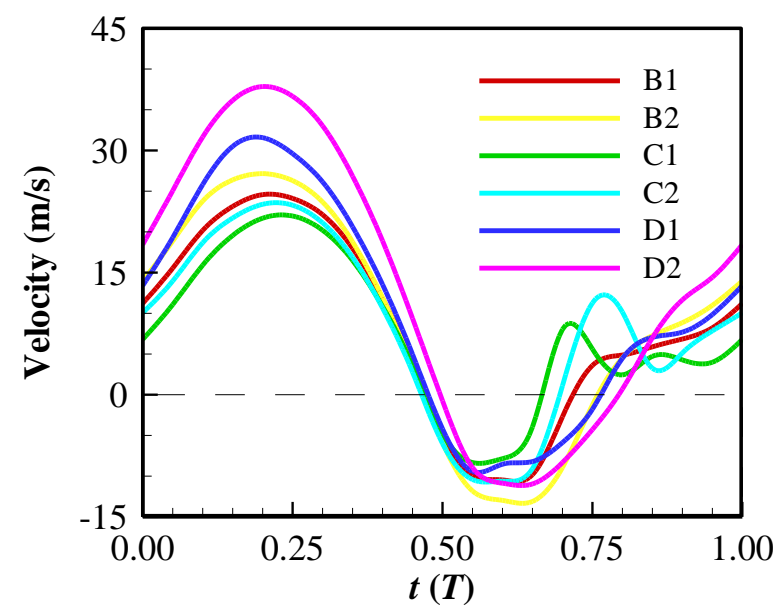

(b)

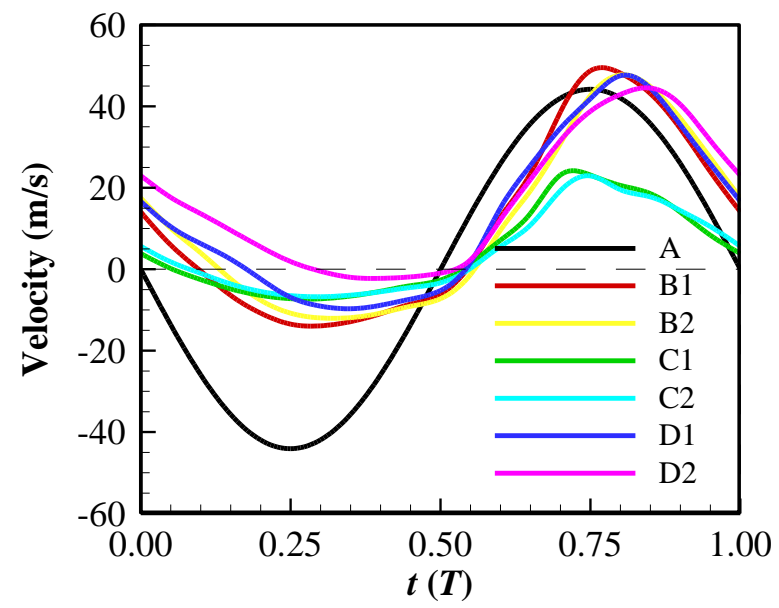

(d)

Fig. 6 Variations of area-averaged velocities with time for different cases: (a) at the orifice (positive: flow downwards); (b) at the inlet (positive: flow in); (c) at the outlet (positive: flow out); and (d) at the nozzle exit (positive: flow downwards).

As shown in Fig. 7(a), the amplitude of the negative volume flow rate and the corresponding time span in one cycle for all the cases based on fluid diodes are less than that for Case A. Meanwhile, the situation is inverse for the positive volume flow rate. The increase of the amplitude is attributed to the supplement of fresh air via the inlet during 
507 ejecting. Compared to Case A, the air volume sucked into the nozzle from the channel is 508 smaller while the air volume ejected from the nozzle is larger for the other cases in one cycle.

509 Among all cases, Case D2 exhibits the least air volume sucked into the nozzle and the 510 maximum time span of the positive volume flow rate in one cycle. Case D2 almost achieves

511 the unidirectional downward flow in the convergence nozzle during one cycle. It should be 512 noted that the air is heated before being sucked into the nozzle, as shown in Fig. 4(a) and Fig.

513 5. The air volume sucked into the nozzle from the channel determines the ejected air 514 temperature while the air volume ejected from the nozzle determines the convective heat 515 transfer ability. The both determine the eventual impingement cooling performance. In order 516 to better evaluate the ability of all cases to reduce the amount of sucked heated air and 517 increase the amount of ejected air via the nozzle exit, the volumetric efficiencies for all cases 518 are calculated according to Eq. (10), which are summarized in Fig. 7(b). The descending 519 order of the volumetric efficiencies for all cases is Case D2 > D1 > B2 > B1 > C2 > C1 > A. 520 All the proposed hybrid synthetic jet actuators significantly increase the volumetric efficiency 521 compared with Case A. Especially, the volumetric efficiency for Case A is zero, while that 522 for Case D2 is $95.8 \%$ which is close to 1 . Moreover, the larger $H_{n}$ can obtain higher 523 volumetric efficiency under the same geometry configuration. 
527

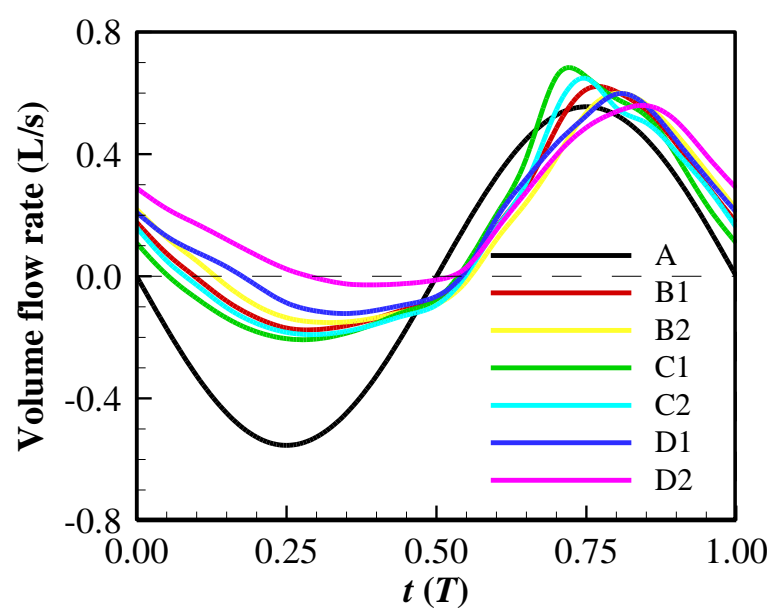

(a)

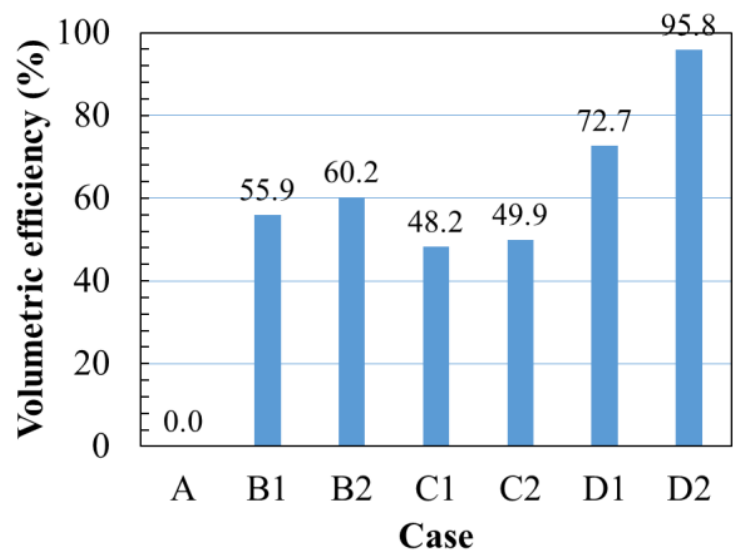

(b)

Fig. 7 (a) Variations of volume flow rate with time via the nozzle exit for all cases (positive: flow downwards); and (b) volumetric efficiency for all cases.

\subsection{Analysis of heat transfer performance}

As previously mentioned, the air is inevitably recirculated within the channel, the nozzle and the cavity at small $H / D_{o}$. Hence, the air in the system is gradually heated. Fig. 8 illustrates the average air temperature evolutions with time at the nozzle exit for all cases. It can be found that the air temperature at the nozzle exit progressively increases and then remains stable after a period of time for all cases. The required time of achieving a stable state increases with the stable temperature. The ascending order of the stable temperature and the required time for all cases is Case $\mathrm{D} 2<\mathrm{D} 1<\mathrm{B} 2<\mathrm{B} 1<\mathrm{C} 2<\mathrm{C} 1<\mathrm{A}$. Among all cases,

Case A has the highest stable air temperature at the nozzle exit and needs the longest time to achieve a stable state. Those are about $56.3{ }^{\circ} \mathrm{C}$ and $2.5 \mathrm{~s}$, respectively. In contrast, Case D2 has the lowest stable air temperature at the nozzle exit and needs a shorter time to achieve a stable state among all cases, which are about $28.7^{\circ} \mathrm{C}$ and $0.8 \mathrm{~s}$. The stable air temperature at the nozzle exit for Case D2 is decreased by $27.6{ }^{\circ} \mathrm{C}$ compared to Case A and only increased by $3.7^{\circ} \mathrm{C}$ compared to the ambient air temperature. The temperature distributions within the computational domain for all cases at $t=2.5 \mathrm{~s}$ are illustrated in Fig. 9. The temperature distributions for all cases are relatively stable $t=2.5$. For Case A, the air temperature within 
548 the cavity above the heated surface increases to $55-60{ }^{\circ} \mathrm{C}$ and that within the channel 549 increases to $55-70{ }^{\circ} \mathrm{C}$. It means that the heated surface is 'cooled' by very hot air. After 550 introducing fluid diodes, the air temperature within the cavity is decreased below $35^{\circ} \mathrm{C}$ and 551 that within the channel above the heated surface is decreased below $40-50{ }^{\circ} \mathrm{C}$ as shown in 552 other cases. Especially for Case D2, the air temperature within the cavity is decreased to 25 $553{ }^{\circ} \mathrm{C}$ and that within the channel above the heated surface is decreased to about $30-45^{\circ} \mathrm{C}$.

554

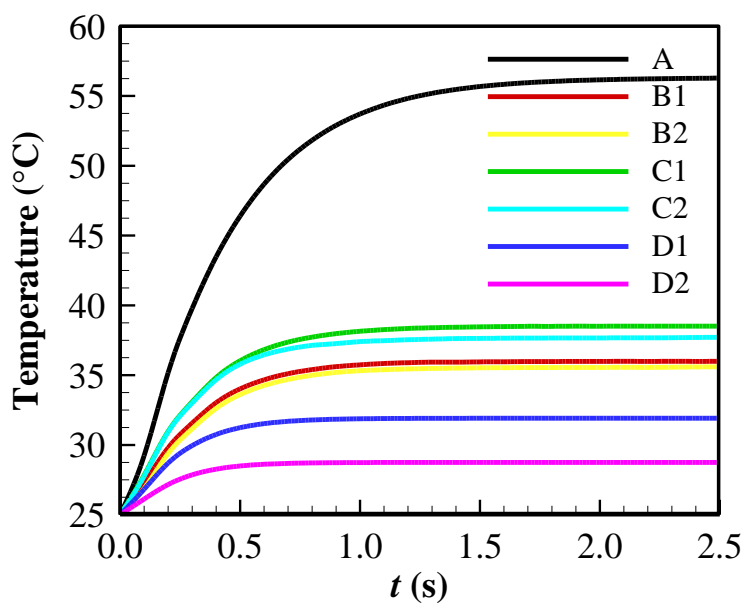

Fig. 8 Average air temperature evolutions with time at the nozzle exit for all cases. 

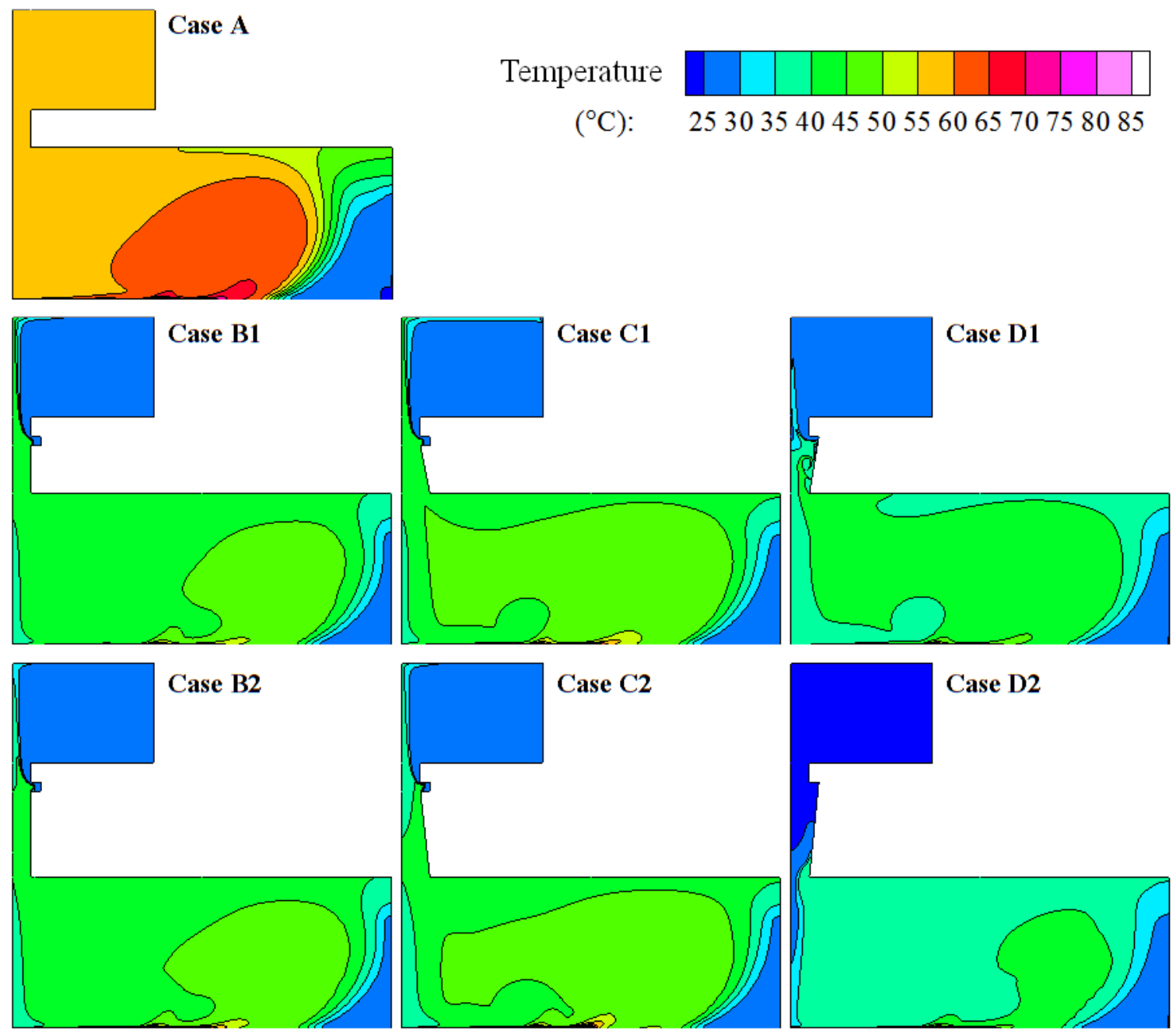

Fig. 9 The temperature distributions within the computational domain for all cases at $t=2.5 \mathrm{~s}$.

The temperature distribution on the heated surface is a crucial indicator for

562 impingement cooling performance of synthetic jets. Fig. 10(a) demonstrates time-averaged

563 temperature profiles on the heated surface at the quasi-stable state $(t=2.5 \mathrm{~s})$. It can be seen

564 that the temperatures on the heated surface for all the cases based on fluid diodes are

565 significantly reduced compared with Case A. Among them, Case D2 achieves the greatest temperature reduction, where the temperatures on the heated surface are decreased to 37.076.9 ${ }^{\circ} \mathrm{C}$ from $65.4-103.9{ }^{\circ} \mathrm{C}$ in Case A. The decreased temperatures ensure the safe and high-

568 efficiency operation of electronic devices. To more clearly compare the cooling performance among all cases, the area-weighted average temperatures on the heated surface for all cases are illustrated in Fig. 10(b). The ascending order of the average temperatures on the heated 
571 surface for all cases is Case D2 $<\mathrm{D} 1<\mathrm{B} 2<\mathrm{B} 1<\mathrm{C} 2<\mathrm{C} 1<\mathrm{A}$. It is obvious that all the

572 hybrid synthetic jet actuators based on fluid diode (Cases B1-D2) offer preferable

573 impingement cooling performance compared to conventional synthetic jet actuator (Case A).

574 The hybrid synthetic jet actuator with a convergent nozzle (Cases D1-D2) exhibits better 575 impingement cooling performance than those with a cylindrical nozzle (Cases B1-B2) and a 576 divergent nozzle (Cases C1-C2). By comparing Cases D1 and D2, it is evident that the larger 577 nozzle length $\left(H_{n}\right)$ makes the advantages of hybrid synthetic jets more pronounced.

578 Compared to Case A, the average temperature on the heated surface for Case D2 decreases 579 by $24.9^{\circ} \mathrm{C}$. Further, the average Nusselt numbers of synthetic jet impinging on the heated 580 surface for all cases, calculated according to Eq. (11), are summarized in Fig. 10(c). The 581 descending order of the average Nusselt numbers for all cases is Case D2 > D1 > B2 > B1 > 582 C2 $>$ C1 > A. Similarly with respect to Case A, Case D2 obtains the maximum heat transfer 583 enhancement among all the cases based fluid diodes, which increases the average Nusselt 584 number by 54.7\%. Based on the combination of Fig. 7(b), Fig. 10(b) and Fig. 10(c), it can be 585 found that the impingement cooling performance and heat transfer ability of synthetic jets are 586 improved as the volumetric efficiency increases. 
590

591

592

593

594

595

596

597

598

599

600

601

602

603

604

605

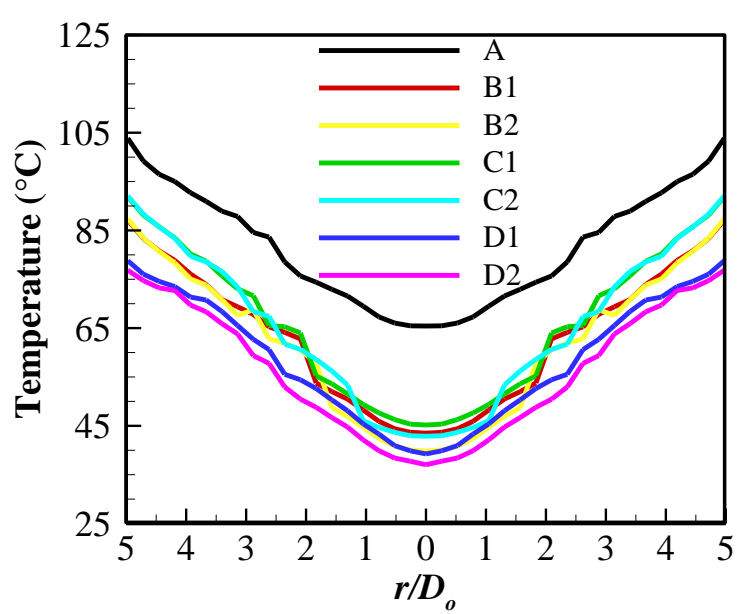

(a)

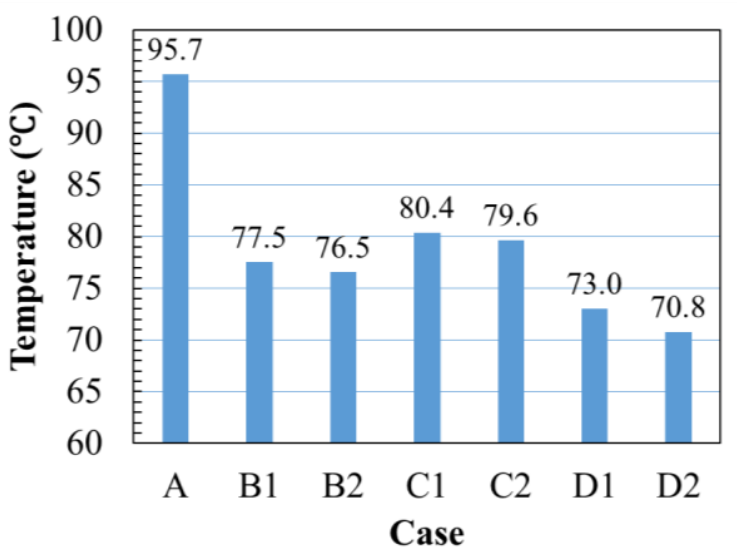

(b)

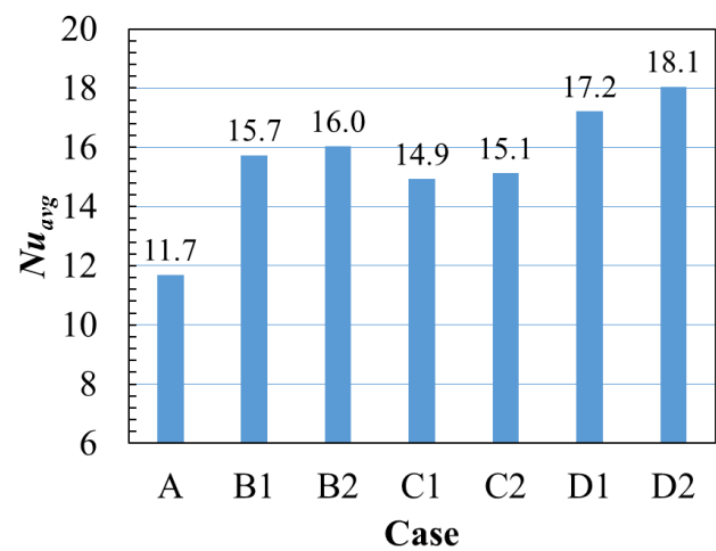

(c)

Fig. 10 Heat transfer data on the heated surface for all cases at the quasi-stable state $(t=2.5 \mathrm{~s}$ ): (a) timeaveraged temperature profiles; (b) area-weighted average temperatures; (c) average Nusselt numbers.

\section{Conclusions}

Various hybrid synthetic jets based on novel fluid diodes are proposed and their performances are numerically analyzed. The following conclusions can be drawn:

(1) Compared to conventional synthetic jet, the cooling performance improvement of the proposed hybrid synthetic jets is attributed to the increase of the volumetric efficiency, which promotes the supplement of cold fresh air and suppresses the recirculation of the heated air.

(2) The hybrid synthetic jet with a convergence nozzle exhibits a higher volumetric efficiency and preferable impingement cooling ability compared to that with a cylindrical or 
606 divergence nozzle. Such configuration nearly achieves the unidirectional downward flow in 607 the nozzle during one cycle and obtains a volumetric efficiency of up to $95.8 \%$.

608 (3) Compared to the conventional synthetic jet, the hybrid synthetic jet with a 609 convergence nozzle decreases the average temperature on the heated surface from $95.7^{\circ} \mathrm{C}$ to $61070.8{ }^{\circ} \mathrm{C}$ and increases the average Nusselt number by $54.7 \%$, which is beneficial for the safe 611 and high-efficiency operation of electronic devices with a high power density.

612

\section{Acknowledgement}

614 The authors would like to acknowledge the financial support of the National Natural 615 Science Foundation of China (Grant Nos. 51606135 and 51776142), the Natural Science 616 Foundation of Hubei Province (Grant No. 2016CFB156) and the Engineering and Physical 617 Sciences Research Council (EPSRC) of the United Kingdom (Grant No. EP/N000714/1). 618 


\section{References}

620 [1] H.M. Maghrabie, M. Attalla, H.E. Fawaz, M. Khalil, Impingement/effusion cooling of electronic 621 components with cross-flow, Applied Thermal Engineering, 151 (2019) 199-213.

622 [2] D. Jagannatha, R. Narayanaswamy, T.T. Chandratilleke, Analysis of a Synthetic Jet-Based 623 Electronic Cooling Module, Numerical Heat Transfer, Part A: Applications, 56 (2009) 211-229.

624 [3] H. Rho, Y.S. Jang, S. Kim, S. Bae, T.-W. Kim, D.S. Lee, J.-S. Ha, S.H. Lee, Porous copper625 graphene heterostructures for cooling of electronic devices, Nanoscale, 9 (2017) 7565-7569.

626 [4] C.S. Greco, G. Paolillo, A. Ianiro, G. Cardone, L. de Luca, Effects of the stroke length and nozzle627 to-plate distance on synthetic jet impingement heat transfer, International Journal of Heat and Mass 628 Transfer, 117 (2018) 1019-1031.

629 [5] B. Giachetti, M. Fénot, D. Couton, F. Plourde, Influence of multi-perforation synthetic jet 630 configuration on heat transfer enhancement, International Journal of Heat and Mass Transfer, 125 631 (2018) 262-273.

632 [6] B. Giachetti, M. Fénot, D. Couton, F. Plourde, Influence of Reynolds number synthetic jet 633 dynamic in crossflow configuration on heat transfer enhancement, International Journal of Heat and 634 Mass Transfer, 118 (2018) 1-13.

635 [7] A. McGuinn, D.I. Rylatt, T.S. O’Donovan, Heat transfer enhancement to an array of synthetic air 636 jets by an induced crossflow, Applied Thermal Engineering, 103 (2016) 996-1003.

637 [8] M. Arik, An investigation into feasibility of impingement heat transfer and acoustic abatement of 638 meso scale synthetic jets, Applied Thermal Engineering, 27 (2007) 1483-1494.

639 [9] R. Mahalingam, A. Glezer, Design and thermal characteristics of a synthetic jet ejector heat sink, 640 Journal of Electronic Packaging, 127 (2004) 172-177.

641 [10] X. He, J.A. Lustbader, M. Arik, R. Sharma, Heat transfer characteristics of impinging steady and 642 synthetic jets over vertical flat surface, International Journal of Heat and Mass Transfer, 80 (2015) $643 \quad 825-834$.

644 [11] G. Krishan, K.C. Aw, R.N. Sharma, Synthetic jet impingement heat transfer enhancement - A 645 review, Applied Thermal Engineering, 149 (2019) 1305-1323.

646 [12] T. Persoons, T.S. O’Donovan, A pressure-based estimate of synthetic jet velocity, Physics of 647 Fluids, 19 (2007) 128104.

648 [13] Q. Gallas, R. Holman, T. Nishida, B. Carroll, M. Sheplak, L. Cattafesta, Lumped Element 649 Modeling of Piezoelectric-Driven Synthetic Jet Actuators, AIAA Journal, 41 (2003) 240-247.

650 [14] T. Persoons, General Reduced-Order Model to Design and Operate Synthetic Jet Actuators, 651 AIAA Journal, 50 (2012) 916-927.

652 [15] Y. Zhang, P. Li, Y. Xie, Numerical investigation of heat transfer characteristics of impinging 653 synthetic jets with different waveforms, International Journal of Heat and Mass Transfer, 125 (2018) 654 1017-1027. 
[16] M. Chaudhari, B. Puranik, A. Agrawal, Heat transfer characteristics of synthetic jet impingement cooling, International Journal of Heat and Mass Transfer, 53 (2010) 1057-1069.

657 [17] M. Chaudhari, B. Puranik, A. Agrawal, Effect of orifice shape in synthetic jet based impingement cooling, Experimental Thermal and Fluid Science, 34 (2010) 246-256.

659 [18] M. Chaudhari, B. Puranik, A. Agrawal, Multiple orifice synthetic jet for improvement in 660 impingement heat transfer, International Journal of Heat and Mass Transfer, 54 (2011) 2056-2065.

661 [19] A. Lee, G.H. Yeoh, V. Timchenko, J.A. Reizes, Heat transfer enhancement in micro-channel 662 with multiple synthetic jets, Applied Thermal Engineering, 48 (2012) 275-288.

663 [20] D.I. Rylatt, T.S. O'Donovan, Heat transfer enhancement to a confined impinging synthetic air jet, 664 Applied Thermal Engineering, 51 (2013) 468-475.

665 [21] U.S. Bhapkar, A. Srivastava, A. Agrawal, Proper cavity shape can mitigate confinement effect in 666 synthetic jet impingement cooling, Experimental Thermal and Fluid Science, 68 (2015) 392-401.

667 [22] Y.-H. Liu, S.-Y. Tsai, C.-C. Wang, Effect of driven frequency on flow and heat transfer of an 668 impinging synthetic air jet, Applied Thermal Engineering, 75 (2015) 289-297.

669 [23] Y.-H. Liu, T.-H. Chang, C.-C. Wang, Heat transfer enhancement of an impinging synthetic air jet 670 using diffusion-shaped orifice, Applied Thermal Engineering, 94 (2016) 178-185.

671 [24] Z. Broučková, Z. Trávníček, Visualization study of hybrid synthetic jets, Journal of 672 Visualization, 18 (2015) 581-593.

673 [25] Z. Trávníček, T. Vít, V. Tesař, Hybrid synthetic jets as the nonzero-net-mass-flux synthetic jets, 674 Physics of Fluids, 18 (2006) 081701.

675 [26] Z. Trávníček, V. Tesař, J. Kordík, Performance of synthetic jet actuators based on hybrid and 676 double-acting principles, Journal of Visualization, 11 (2008) 221-229.

677 [27] J. Kordík, Z. Trávníček, Novel Fluidic Diode for Hybrid Synthetic Jet Actuator, Journal of Fluids 678 Engineering, 135 (2013) 101101-101107.

679 [28] Z. Trávníček, T. Vít, Impingement heat/mass transfer to hybrid synthetic jets and other reversible 680 pulsating jets, International Journal of Heat and Mass Transfer, 85 (2015) 473-487.

681 [29] S.-S. Hsu, Z. Travnicek, C.-C. Chou, C.-C. Chen, A.-B. Wang, Comparison of double-acting and 682 single-acting synthetic jets, Sensors and Actuators A: Physical, 203 (2013) 291-299.

683 [30] S.S. Hsu, Y.J. Chou, Z. Trávníček, C.F. Lin, A.B. Wang, R.H. Yen, Numerical study of nozzle 684 design for the hybrid synthetic jet actuator, Sensors and Actuators A: Physical, 232 (2015) 172-182.

685 [31] M. Hatami, F. Bazdidi-Tehrani, A. Abouata, A. Mohammadi-Ahmar, Investigation of geometry 686 and dimensionless parameters effects on the flow field and heat transfer of impingement synthetic 687 jets, International Journal of Thermal Sciences, 127 (2018) 41-52.

688 [32] A. Miró, M. Soria, J.C. Cajas, I. Rodríguez, Numerical study of heat transfer from a synthetic 689 impinging jet with a detailed model of the actuator membrane, International Journal of Thermal 690 Sciences, $136(2019)$ 287-298. 
691 [33] P. Li, D. Guo, R. Liu, Mechanism analysis of heat transfer and flow structure of periodic 692 pulsating nanofluids slot-jet impingement with different waveforms, Applied Thermal Engineering, $693152(2019)$ 937-945.

694 [34] T. Persoons, A. McGuinn, D.B. Murray, A general correlation for the stagnation point Nusselt 695 number of an axisymmetric impinging synthetic jet, International Journal of Heat and Mass Transfer, 69654 (2011) 3900-3908.

697 [35] D. Zhang, K. Yang, H.E. Qu, J. Gao, Numerical Investigation of Heat Transfer Performance of 698 Synthetic Jet Impingement onto Dimpled/protrusioned Surface, Thermal Science, 19 (2015) S221699 S229.

700 [36] M. Jain, B. Puranik, A. Agrawal, A numerical investigation of effects of cavity and orifice 701 parameters on the characteristics of a synthetic jet flow, Sensors and Actuators A: Physical, 165 702 (2011) 351-366.

703 [37] Q. Xia, S. Lei, J. Ma, S. Zhong, Numerical study of circular synthetic jets at low Reynolds 704 numbers, International Journal of Heat and Fluid Flow, 50 (2014) 456-466. 